https://helda.helsinki.fi

\title{
Basis of genetic adaptation to heavy metal stress in the acidophilic green alga Chlamydomonas acidophila
}

\section{Puente-Sánchez, Fernando}

2018-07

Puente-Sánchez , F, Díaz , S, Penacho , V , Aguilera, A \& Olsson , S 2018 , ' Basis of genetic adaptation to heavy metal stress in the acidophilic green alga Chlamydomonas acidophila ' , Aquatic Toxicology , vol. 200 , pp. 62-72 . https://doi.org/10.1016/j.aquatox.2018.04.020

http://hdl.handle.net/10138/314407

https://doi.org/10.1016/j.aquatox.2018.04.020

cc_by_nc_nd

acceptedVersion

Downloaded from Helda, University of Helsinki institutional repository.

This is an electronic reprint of the original article.

This reprint may differ from the original in pagination and typographic detail.

Please cite the original version. 


\section{Basis of genetic adaptation to heavy metal stress in the acidophilic green alga Chlamydomonas acidophila}

Fernando Puente-Sánchez ${ }^{\mathrm{a}}$, Silvia Díaz ${ }^{\mathrm{b}}$, Vanessa Penachoc ${ }^{\mathrm{c}}$, Angeles Aguilerad ${ }^{\mathrm{d}}$ and Sanna Olsson ${ }^{\mathrm{e}, \mathrm{f}^{*}}$ Running title: Genetic adaptation of extremophilic C. acidophila

aSystems Biology Program, Centro Nacional de Biotecnología (CNB-CSIC), Calle Darwin 3, 28049, Madrid, Spain

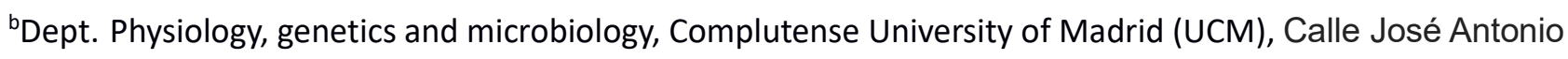
Novais 12, 28040 Madrid, Spain

'Bioarray, S.L. Parque Científico y Empresarial de la UMH. Edificio Quorum III. Avenida de la Universidad s/n, 03202 Elche, Alicante, Spain

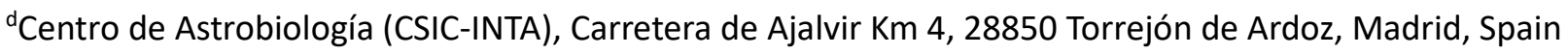
eINIA Forest Research Centre (INIA-CIFOR), Dept. Forest Ecology and Genetics, Carretera de la Coruña km 7.5, 28040 Madrid, Spain

fDept. Agricultural Sciences, P.O. Box 27, 00014 University of Helsinki, Finland

*corresponding author : Sanna Olsson, INIA Forest Research Centre (INIA-CIFOR), Dept. Forest Ecology and Genetics, Carretera de A Coruña km 7.5, E-28040 Madrid, Spain, tel. +34 913476773, fax: +34 913476767, e-mail: sanna.olsson@helsinki.fi 
2 To better understand heavy metal tolerance in Chlamydomonas acidophila, an extremophilic green alga, we

3 assembled its transcriptome and measured transcriptomic expression before and after Cd exposure in this and the neutrophilic model microalga Chlamydomonas reinhardtii. Genes possibly related to heavy metal tolerance and detoxification were identified and analyzed as potential key innovations that enable this species to live in an extremely acid habitat with high levels of heavy metals. In addition we provide a data set of single orthologous genes from eight green algal species as a valuable resource for comparative studies including eukaryotic extremophiles.

Our results based on differential gene expression, detection of unique genes and analyses of codon usage all indicate that there are important genetic differences in C. acidophila compared to C. reinhardtii. Several efflux family proteins were identified as candidate key genes for adaptation to acid environments. This study suggests for the first time that exposure to cadmium strongly increases transposon expression in green algae, and that oil biosynthesis genes are induced in Chlamydomonas under heavy metal stress. Finally, the comparison of the transcriptomes of several acidophilic and non-acidophilic algae showed that the Chlamydomonas genus is polyphyletic and that acidophilic algae have distinctive aminoacid usage patterns.

Key words: heavy metals, transcriptomics, green algae, Río Tinto, extremophiles, transposons

\section{INTRODUCTION}

Cd is a widespread environmental pollutant which is even at low concentrations extremely toxic to aquatic microorganisms, in particular microalgae (Brayner et al., 2011; Wang et al., 2013). In spite of its harmfulness there exist very few studies on transcriptomic alterations caused by increased levels of this or other heavy metals in green algae, (Hutchins et al., 2010; Jamers et al., 2013; Zhang et al., 2014). Cd binds to organic 
molecules by forming bonds with sulfur and nitrogen, thereby inactivating proteins causing a broad range of adverse effects. It is easily absorbed and bio-accumulated by lower organisms and transferred to higher trophic levels in food chain. It has been shown to inhibit growth (Okamoto et al., 1996), chlorophyll and chloroplast synthesis (Lamai et al., 2005), cause disintegration of the cell wall as well as induce a large increase in superoxide dismutase (SOD) activity, indicative of oxidative stress (Okamoto et al., 1996). Additionally, Cd replaces zinc and selenium at the active sites of enzymes, competes with other ions in membrane transport, andecreases RNA and DNA synthesis as well as photosynthetic pigments and proteins (Prasad et al., 1999; Wang et al., 2013).

The extremophilic green alga Chlamydomonas acidophila grows in very acidic environments (pH 2.3-3.4). Metal sequestration in vacuoles seems to be an important mechanism in cadmium tolerance and detoxification in $C$. acidophila (Aguilera and Amils, 2005) but there is evidence that also unique genetic features in C. acidophila contribute to its high heavy metal tolerance (Olsson et al., 2015; Olsson et al., 2017). The strain analyzed in this work was isolated from Río Tinto (SW Spain), one of the most extensive examples of natural extreme acidic environments (Fernández-Remolar et al., 2003). The river has a low pH (ranging from 0.8 to 2.5) buffered by ferric iron and with high concentrations of heavy metals (Aguilera et al., 2006). These extreme conditions are produced by the metabolic activity of chemolithotrophic prokaryotes that are found in high numbers in its waters (González-Toril et al., 2003). Despite these extreme environmental conditions, Río Tinto shows an unexpected degree of eukaryotic diversity (Amaral-Zettler et al., 2011). Cd was chosen for this study due to its toxicity and also because it is found in very high concentrations in Río Tinto, with local average amounts that can reach ca. 40 mg/L (Aguilera et al., 2007).

Research on extremophilic organisms significantly contribute to our understanding of the evolution of heavy metal tolerance in plants and algae. The results enable detection of novel genes potentially useful for 
biotechnology and phytoremediation of contaminated water resources. In spite of this, there is very limited

genetic data available for $C$. acidophila while the genome of $C$. reinhardtii has been sequenced and annotated (Merchant et al., 2007; Manichaikul et al., 2009). For C. reinhardtii, there also exist several physiological, molecular, and genetic studies including experimental verification of the functionality of the predicted ORFs (Ghamsari et al., 2011). To increase genomic resources in C. acidophila we assembled an improved transcriptome for this non-model species. We compared it to the transcriptomes of the model microalga Chlamydomonas reinhardtii from the same genus and other publicly available green algal transcriptomes. To explain how $C$. acidophila is able to survive extreme environments we used transcriptomic sequencing and qRTPCR to detect transcriptional changes caused by high Cd concentrations in C. reinhardtii and C. acidophila and identified possible adaptive key genes. The high level of genes with unknown function as well as lack of an annotated genome assembly makes the identification of important genes involved in heavy metal detoxification in C. acidophila challenging. In spite of these difficulties we provide new information on heavy metal tolerance in this organism, extremophiles and green algae in general.

\section{MATERIAL AND METHOdS}

\subsection{Sample collection, cultivation and exposure to Cadmium}

Chlamydomonas acidophila strain RT46 was collected from water samples taken in 2010 at the CEM sampling station of Río Tinto (SW Spain) (Aguilera et al., 2006), and isolated to grow in the presence of antibiotics, vancomycin $50 \mu \mathrm{g} / \mathrm{mL}$, cefotaxime $100 \mu \mathrm{g} / \mathrm{mL}$ and chloramphenicol $15 \mu \mathrm{g} / \mathrm{mL}$ (Sigma), on agar plates made with $0.22 \mu \mathrm{m}$-filtered river water. Individual colonies were transferred into K medium (Keller et al., 1987), pH 2. A strain of Chlamydomonas reinhardtii (CC-1374, SAG 77.81) was purchased from the Chlamydomonas Resource Center (University of Minnesota) and grown in $\mathrm{K}$ medium, $\mathrm{pH}$ 7. The $\mathrm{K}$ medium was supplied with the same antibiotics as the ones used for cell isolation (vancomycin $50 \mu \mathrm{g} / \mathrm{mL}$, cefotaxime $100 \mu \mathrm{g} / \mathrm{mL}$ and chloramphenicol $15 \mu \mathrm{g} / \mathrm{mL}$ ). 
The algae were grown under ca. $70 \mu \mathrm{E} \mathrm{s}^{-1} \mathrm{~m}^{-2}$ irradiance provided by day-light fluorescent tubes, 16:8 h LD cycle and $22{ }^{\circ} \mathrm{C}$ of temperature. The cultivations were refreshed every two weeks in corresponding growth media and cells undergoing exponential growth were grown to be treated with metalloid solutions. To reach exponential growth $5 \mathrm{ml}$ of Chlamydomonas cultivate was transferred into an $1 \mathrm{~L}$ Erlenmeyer bottle with $500 \mathrm{ml}$ medium. After 10 days of growth $15 \mathrm{ml}$ of cultivate was transferred into three $2 \mathrm{~L}$ Erlenmeyer bottles with $980 \mathrm{ml}$ medium in each.

For the transcriptomic sequencing a Cd solution $\left(\mathrm{CdCl}_{2} \times 2 \frac{1 / 2}{2} \mathrm{H}_{2} \mathrm{O}\right)$ with a final concentration of $245 \mu \mathrm{M}$ was used. Earlier studies on Chlamydomonas showed a peak of gene expression between three and four hours in genes involved in cadmium tolerance (Hanikenne et al., 2005; Olsson et al. 2017). Therefore time points for cell collection were set before the treatment, at $3 \mathrm{~h}$ and $6 \mathrm{~h}$ after $\mathrm{Cd}$ exposure. The cells were collected in $50 \mathrm{ml}$ Falcon tubes, centrifuged for $10 \mathrm{~min}$ in $5000 \mathrm{rpm}$, the supernatant was discarded and the pellets frozen at $-80{ }^{\circ} \mathrm{C}$ until RNA extraction. For qRT-PCR cultures were treated with following solutions: $1 \mu \mathrm{M} \mathrm{Cd}$ solution $\left(\mathrm{CdCl}_{2} \times 2 \frac{1}{2} \mathrm{H}_{2} \mathrm{O}\right)$, $1 \mathrm{mM} \mathrm{Cu}\left(\mathrm{CuSO}_{4} \times \mathrm{HH}_{2} \mathrm{O}\right), 10 \mathrm{mM} \mathrm{Fe}\left(\mathrm{FeSO}_{4} \mathrm{X} 7 \mathrm{H} 2 \mathrm{O}\right), 1 \mathrm{mM}$ As (III) $\left(\mathrm{AsNaO}_{2}\right)$ or $5 \mathrm{mM} \mathrm{As}(\mathrm{V})\left(\mathrm{Na}_{2} \mathrm{HAsO}_{4}\right)$ and cells were collected at 1, 3 or $24 \mathrm{~h}$ after exposure.

\subsection{RNA extraction and sequencing}

Total RNA was extracted with TRI Reagent ${ }^{\circledR}$ Solution (Ambion, Life Technologies, CA, USA) following manufacturer's protocol. RNA quality and quantity were estimated using an Agilent 2100 bioanalyzer (Agilent Technologies). RNA library preparation and high-throughput sequencing were carried out in the NGS sequencing Unit (Scientific Park Foundation, Madrid, Spain) using Illumina GAiix sequencing platform. One full lane of 75 basepair long reads for each sample was sequenced to provide sufficient coverage for a representative overview of the expression profile. The generated transcriptome library was non-normalized to allow detection of differences on the gene expression level between the different treatments and untreated cultures. 


\subsection{Data preprocessing de novo hybrid assembly}

All raw transcriptomic reads were filtered and trimmed with PRINSEQ lite (version 0.18 .3 (Schmieder and Edwards, 2011) in order to remove duplicates and low quality reads (using default parameters except for the following: -min qual mean 25 -derep 12, -ns max p 1 -derep 12 -lc method dust -Ic threshold 7 -trim tail left 6 trim tail right 6 -trim ns left 2 -trim ns right 2 -trim qual left 25 -trim qual right 25 ).

The single-end Illumina reads from $C$. acidophila obtained in this study were combined with the 454 reads obtained in Olsson et al., (2015). Paired-end Illumina reads were simulated from 454 reads by using the run_simulate_illuminaPE_from_454ds.sh script included in the Trinity suite. The resulting reads were subsequently normalized in silico with the normalize_by_kmer_coverage.pl included in Trinity. The paired-end normalized reads coming from the 454 dataset were pooled together with the single-end Illumina reads obtained in this study, and assembled with Trinity (release 2013_08_14) (Grabherr et al., 2011) using Jellyfish (Marcais and Kingsford, 2011) for k-mer counting with a maximum memory of 40G, minimum contig length of 200, paired fragment length of 350 and a maximum butterfly heap space of 20G. Contigs with a BLASTn identity of more than $90 \%$ to the E. coli, C. reinhardtii and human transcriptomes were discarded.

\subsection{Abundance estimation and transcriptome coverage analysis}

The RSEM software package (version 1.1.18.modified) (Li and Dewey, 2011) was used to estimate the gene and isoform expression values. For C. acidophila, a reference transcriptome was generated from the Trinity assembly by using the RSEM commands extract-transcript-to-gene-map-from-trinity and rsem-prepare-reference with default parameters. For $C$. reinhardtii, the reference transcriptome v4.0 (Merchant et al., 2007) available from Phytozome (http://www.phytozome.net/) was used as a reference for estimating transcript expression. Reads from the six samples were aligned separately to the reference transcriptomes by using Bowtie (version 0.12.7) (Langmead et al., 2009) and expression values for each sample were obtained with RSEM. The resulting 
121

expression counts were normalized with the trimmed mean of M-values method, as implemented in the edgeR package (version 2.15.0) (Robinson et al., 2010). The transcripts with a log2 fold change higher than 6 and FPKM (Fragments Per Kilobase Million) of more than 20 in at least one sample were selected for further analysis. For $C$. acidophila, only the longest transcript per Trinity subcomponent was reported.

In order to assess the coverage of each sequence in our $C$. acidophila assembly, reads from the three $C$. acidophila samples were pooled and aligned against the reference transcriptome. We used the align-reads.p/ script included in the Trinity package (release 2013_08_14), resorting to Bowtie (version 0.12.7) to perform the alignment. The script also utilized Samtools (version 0.1.18) (Li et al., 2009) for SAM-format alignment manipulations. The output file bowtie out.coordSorted.bam, which contains both properly mapped pairs and single unpaired fragment reads, was used as input for Qualimap (version 0.6) (García-Alcalde et al., 2012) in order to estimate transcript coverage.

\subsection{Taxonomic and functional annotation}

All transcripts were annotated via BLASTx searches (Altschul et al., 1997). For taxonomic annotation GenBank's non-redundant protein database (nr) was used. For functional annotation two other major databases, Uniprot's Swiss-Prot and TrEMBL protein databases were used in addition to the nr database to get more accurate information on genetic functions. Taxonomic and functional information from the multiple databases for each differentially expressed contig was collected into a table preferring the most accurate functional annotation from Swiss-Prot when available using the methods and scripts modified from de Wit et al., (2012).

\subsection{Protein prediction and orthology search with OrthoFinder across green algal transcriptomes}

To identify orthologous gene groups among green algae, representative transcriptome files were downloaded from Phytozome v.11 (http://www.phytozome.net/) for six available species: Chlamydomonas reinhardtii, 
Coccomyxa subellipsoidea, Micromonas pusilla, Micromonas sp. RCC299, Osterococcus lucimarinus and Volvox carteri. The de novo assemblies of $C$. acidophila and D. acidophila (Puente-Sánchez et al., 2016) were translated to amino acids with TransDecoder (v. 3.0.0, The Broad Institute). Orthologous sequences from these eight species were grouped with the clustering software OrthoFinder (Emms and Kelly, 2015). The resulting alignments were filtered to contain only the longest isoform of $C$. acidophila and $D$. acidophila when several isoforms of the same gene (belonging to same component and subcomponent in the de novo assembly built were subject to further analyses while orthologous groups representing putative single-copy nuclear genes (an orthologous group with exactly one gene / species) present in all species were used to build a phylogeny.

\subsection{Genes present in C. acidophila but not in C. reinhardtii}

To find an explanation for the different responses to heavy metals in extremophiles and neutrophiles, two approaches to identify genes that are present in C. acidophila but not C. reinhardtii were employed. First, screening for genes related to heavy metal tolerance and detoxification was done based on keywords in the annotation of the $C$. acidophila transcriptome. Only transcripts that had other organisms than $C$. reinhardtii as first BLAST match were included. To verify that the identified candidate genes are not present in C. reinhardtii, a local BLASTn search against C. reinhardtii transcripts was performed. Reciprocal BLAST was performed to confirm the matches and confirmed isoforms were used in downstream analyses.

Secondly, to identify genes specific to acidophiles, orthologous groups containing both of the extremophiles ( $C$. acidophila and $D$. acidohila) but not $C$. reinhardtii were extracted. As a precaution to exclude contaminant sequences, in the absence of reference genomes for the extremophiles, only the orthologous groups containing at least one additional green algal species were kept. In addition transcripts with organellar annotations (mitochondrial or chloroplast) were excluded. Phylogenetic analyses were made for the transcripts with an 
annotation related to heavy metal tolerance and detoxification after confirming their absence in $C$. reinhardtii by a BLASTx against nr database with a cut-off E-value of $\leq 10-3$.

171

172

\subsection{Phylogenetic analyses}

173 Sequences were aligned with Mafft (Katoh et al., 2002). For individual genes the alignments were manually 174 edited in PhyDE ${ }^{\circledR}$ v1.0 (Müller et al., 2005) by excluding ends of the alignments which could not be confidently 175 aligned due to length differences and ambiguities in homology assessment. The concatenated data matrix of 488 single orthologous groups was trimmed with Trimal (Capella-Gutierrez et al., 2009) using the option gappyout. Bayesian analyses were performed with MrBayes v3.2.1 (Ronquist et al., 2012), applying the suggested search strategies for amino acids (Huelsenbeck et al., 2001; Ronquist and Huelsenbeck, 2003). For the individual genes four runs with four chains $\left(1 \times 10^{6}\right.$ iterations each) were run simultaneously while for the concatenated matrix of 488 single orthologous groups four runs with two chains $\left(1 \times 10^{6}\right.$ iterations each) were run. Chains were sampled every 1000 generations and the respective trees written to a tree file. Calculations of the consensus tree and of the posterior probability of clades were performed based upon the trees sampled after the chains converged. The concatenated matrix was also analyzed using RAxML (Stamatakis, 2006;

Stamatakis et al., 2008) defining the used model automatically with the option -m PROTGAMMAAUTO.

Consensus topologies and support values from the different methodological approaches were compiled and drawn using TreeGraph2 (Stöver and Müller, 2010).

\subsection{Quantitative reverse transcription PCR (qRT-PCR)}

For qRT-PCR protocols established by Díaz et al., (2007) were followed, applying the modifications detailed in

Olsson et al., (2017). Actin (ACT1) and 18S were used as endogenous control genes. All qRT-PCR reactions were carried out in an iQTM5 multicolor Real-Time PCR detection System (Bio-Rad) apparatus with the following 
$\mathrm{s}$ and $72^{\circ} \mathrm{C}$ after $20 \mathrm{~s}$. Both NTC (no template control) and RT minus control were negative. The real-time dissociation curve was used to check primer specificity and to confirm the presence of a unique PCR product. Standard curves were obtained using 10-fold serial cDNA dilutions and determining the Ct (cycle threshold) values. The standard line parameters (amplification efficiency, slope and correlation coefficient) are reported in Table 1. Analysis of relative gene expression was carried out according to the Standard-curve quantification method (Larinov et al., 2005) from, at least, four independent experiments (each performed in duplicates). Primers for qRT-PCR were designed using the program Primer3 (http://frodo.wi.mit.edu/cgibin/primer3/primer3_www.cgi) with default settings. All primers used in this study are listed in Table 2.

\subsection{Codon usage bias and GC content analyses}

Complete CDSs (coding DNA sequences) were extracted from the eight algal transcriptomes (Chlamydomonas acidophila, Dunaliella acidophila, Chlamydomonas reinhardtii, Coccomyxa subellipsoidea, Micromonas pusilla, Micromonas sp. RCC299, Osterococcus lucimarinus and Volvox carteri) by using the Transdecoder software included in the Trinity suite. GC content and codon and aminoacid usage for each CDS were calculated with GCUA (General Codon Usage Analysis; Mclnerney, 1998). For each gene, only the longest transcript was included in the analysis.

\subsection{GO-terms enriched/depleted in particular aminoacids in acidophiles versus non-acidophiles}

211 In order to detect Gene Ontology (GO) terms with a significant enrichment/depletion of particular aminoacids in acidophiles versus non-acidophiles, the following procedure was followed. Firstly, we selected the proteins with i) less than $2 \%$ of glutamate, ii) less than $2 \%$ of aspartate, iii) more than $4 \%$ of cysteine, iv) more than $15 \%$ of serine. These proteins will henceforth be referred to as "extreme" proteins. The particular aminoacids and the percentage cutoff values were selected after inspection of the aminoacid utilization profiles shown in the 
217 counted the number of appearances of each GO-term in the "extreme" proteomes and in the "non-extreme" 218 proteomes of the two acidophilic species and the six non-acidophilic species, respectively. This was information used to build the following contingency table for each GO-term, which was subjected to the Fisher's Exact test in order to assess whether that particular GO-term was significantly enriched $(p<0.05)$ in the extreme fraction of the proteome in acidophiles versus non-acidophiles, that is, was significantly enriched/depleted in that particular aminoacid in acidophiles versus non-acidophiles.

223

224

\begin{tabular}{|c|c|}
\hline $\begin{array}{c}\text { \# GO-term appearances in the extreme proteome of } \\
\text { acidophiles }\end{array}$ & $\begin{array}{c}\text { \# GO-term appearances in the non-extreme proteome } \\
\text { of acidophiles }\end{array}$ \\
\hline $\begin{array}{c}\text { \# GO-term appearances in the extreme proteome of } \\
\text { non-acidophiles }\end{array}$ & $\begin{array}{c}\text { \# GO-term appearances in the non-extreme proteome } \\
\text { of non-acidophiles }\end{array}$ \\
\hline
\end{tabular}

3 RESULTS AND DISCUSSION

\subsection{High-throughput sequencing, assembly and taxonomic annotation of $C$. acidophila transcripts}

Six single-end Illumina Hi-Seq libraries were sequenced in order to monitor the transcriptomic response of

Chlamydomonas reinhardtii and Chlamydomonas acidophila to cadmium stress right after cadmium exposure, three hours after exposure and six hours after exposure. A total of 131,128,472 raw reads were generated, of which $66,677,308$ passed quality filtering, with the duplication level being consistent with that found in other studies (Gómez-Álvarez et al., 2009).

In order to obtain a high quality draft transcriptome for $C$. acidophila, the reads obtained in this study were pooled together and co-assembled with the reads obtained in Olsson et al., (2015). This yielded 151449 transcripts of unique isoforms corresponding to 47411 unique Trinity subcomponents (which can be interpreted as distinct genes), with a N50 of 3212 nucleotides, and average isoform coverage of 54.62X. The pre-processing 
and assembly statistics are summarized in Tables 3A and 3B. The hybrid assembly significantly improved the assembly results and genome fraction coverage over the existing assembly from the earlier study (GenBank accession GBAH00000000) for which only 454 reads were used.

241

\subsection{Differential expression analysis of transcripts}

For both species, the gene expression after $3 \mathrm{~h}$ and $6 \mathrm{~h}$ of cadmium exposure was compared to the gene expression right before cadmium exposure. H43 (Rubinelli et al., 2002) and Cds1 (Hanikenne et al., 2005) are among the few genes that have been identified to be induced by cadmium in $C$. reinhardtii. In addition a novel phytochelatin synthase CaPCS2 was recently showed to be strongly induced by $\mathrm{Cd}$ in C. acidophila (Olsson et al., 2017). Transcripts homologous to these genes were not found to be differentially expressed in this study, possibly due to the strict cutoff values applied. The time and concentration of the exposure might also greatly affect the transcriptomic response of green algae to Cd. Hanikenne et al., (2005) observed a peak of expression in the half-size $A B C$ transporter gene $C d s 1$ at 4 hours after 200-400 $\mu \mathrm{M}$ Cd exposure and argued that the transcript levels of this gene were too low to be detected under the experimental conditions ( $2 \mathrm{~h}$ exposure to $25 \mu \mathrm{M}$ cadmium) used earlier by Rubinelli et al. (2002). On the other hand, Olsson et al., (2017) reported a very strong induction of the gene CaPCS2 in as low concentration as $1 \mu \mathrm{M}$. Furthermore, different isoforms might result in different expression values.

In this study we focused on genes showing differential gene expression when exposed to very strong Cd exposure. To complement the gene expression profile of selected candidate genes qRT-PCR was performed using different concentrations and time points.

\subsection{Differentially expressed genes in C. reinhardtii}


261 The low number of transcripts detected to be differentially expressed in C. reinhardtii (Additional File 1) is likely

262 due to the high amount of $\mathrm{Cd}$ used in the experiment, which was chosen to give a visible effect on the transcriptomic expression in C. acidophila.

264

The transcripts with highest increase in expression after $\mathrm{Cd}$ exposure between control and one of the cadmium treated samples include transcripts coding for an apoptosis-inducing factor, NSG6 protein, NifU-like protein 5, and a vacuolar protein sorting-associated protein, in addition to transcripts with unknown function. Induction of stress related genes, as well as genes operating in metal uptake and export as a response to cadmium has been observed previously in C. reinhardtii (Jamers et al., 2013) as well as in cyanobacteria (Houot et al., 2007).

Other differentially expressed genes could not be directly linked to heavy metal detoxification but can nitrogen starvation (Abe et al., 2004). Interestingly, gametogenesis in C. reinhardtii leads to an increased production of lipids with use as biofuels (Miller et al., 2010). Here we show that, apart from nitrogen starvation, this process can also be induced by heavy metal stress, opening the way for novel engineering strategies in the search for high oil yields

\subsection{Differentially expressed genes in C. acidophila}

The top fifty up regulated higher transcripts are summarized in Additional File 2, and included several transposable elements. Significant upregulation was observed in a transcript annotated as retrotransposon copia (FPKM in 0h 0, 3h 240.83, 6h 840.66 in comp17295_c0_seq16), a transcript annotated as retrovirusrelated Pol polyprotein from transposon TNT 1-94 (the annotation varies according to isoform, highest FPKM in comp17071_c1_seq27: 0h 1.84, 3h 156.11, 6h 676.71), retrovirus-related Pol polyprotein from transposon 297 (comp18064_c0_seq15) and Transposon Ty3-I Gag-Pol polyprotein (comp16440_c0_seq16). Retrotransposons 
regulation in plants (Flavell et al. 1992), some being transferred horizontally (Cheng et al. 2009 and references therein). There are indications that retrovirus and retrotransposons are involved in gene regulation and detoxification of heavy metals. Retrovirus-related Pol polyprotein from transposon TNT 1-94 has been shown to alter its methylation status in Populus alba when grown on heavy metal contaminated soil (Cicatelli et al., 2014). Castrillo et al., (2013) showed that heavy-metal stress induced transposon activity in plants. Exposure to Cd strongly increased transposon expression in C. acidophila, which suggests for the first time that heavy-metal stress induces transposon activity also in green algae.

There are several transcripts with the annotation arsenite resistance protein ArsB among the differentially expressed genes (comp14907_c0 or comp15936_c0). The annotations are partly incongruent, comp14907_c0 getting annotated as arsenite resistance protein ArsB or ubiquitin-like modifier-activating enzyme ATG7, while the annotations for comp15936_c0 are arsenite resistance protein ArsB or arsenate reductase. The automated annotation is complicated by the fact that the nomenclature of the ACR3 family ArsB protein overlaps with ArsB of E.coli belonging to the ArsB family (Wu et al., 1992). It was verified from the alignments including all isoforms (data not shown) that all isoforms of one component belong together, and the different annotations are due to lack of highly similar sequences in GenBank of some sequence parts. To avoid confusions in this manuscript the isoforms of comp14907_c0 are referred to arsenical-resistance protein ACR3 and isoforms of comp15936_c0 as ACR3 family arsenite transporter based on the annotation of the consensus sequences of these isoforms. Most differentially expressed transcripts of both comp15936_c0 and comp14907_c0 are strongly induced by Cd, with the exception of comp15936_c0_seq52. However, according to qRT-PCR analyses the ACR3 family arsenite transporter comp_15936_c0 is down-regulated by Cd (Table 4). The incongruent results between the measures based on the gene expression data and the qRT-PCR are likely due to the differences in the used Cd concentrations. 
An oil globule associated protein (comp13235_c0_seq1) was detected to be induced by copper by Olsson et al.,

310 (2015), and is now shown to be also induced by Cd (FPKM in 0h 0, 3h 10.058, $6 \mathrm{~h}$ 30.682). This again highlights the role of heavy metals as inductors of oil production in Chlamydomonas (see previous section). Furthermore, the ability of $C$. acidophila to tolerate extreme acidity and heavy metal concentrations might help it avoid the contamination issues that commonly hamper microalgal biodiesel production (Siaut et al., 2011; Wang et al., 2016). While a detailed study of the oil production potential of $C$. acidophila is beyond the scope of this manuscript, our findings warrant further investigation on its biotechnological applications.

\subsection{Species phylogeny based on orthologous sequences}

We identified 488 single orthologues present in all eight species (Additional File 3), which were used to build a species phylogeny. According to the phylogenomic analyses the genus Chlamydomonas is not monophyletic

(Fig. 1). This is not so surprising since Chlamydomonas is known to be polyphyletic and in need for revision, first shown by Buchheim et al., (1990) and confirmed by several later studies (e.g. Leliaert et al., 2012; Nakada et al., 2016). However, earlier phylogenies have been based on few molecular markers and now the polyphyly of Chlamydomonas is shown for the first time on a phylogenomic level. Micromonas pusilla was resolved as best root in the species tree. All clades got full support both with MrBayes and RAxML.

\subsection{Identification of genes unique to C. acidophila}

Some genes can be important in heavy metal tolerance and metal homeostasis even if their expression is not altered in the presence of the metal. Most phytochelatin synthases, for example, are known to be constitutively expressed but post-translationally activated by heavy metals in plants (Cobbett and Goldsborough, 2002; Rea et al., 2004). To better understand the mechanisms that enable $C$. acidophila to live in its extremely acid environment we therefore identified genes involved in heavy metal tolerance and detoxification that are 
332

333

334

335

336

337

338

339

340

341

342

343

344

345

346

347

348

349

350

351

352

353

354

355

present in C. acidophila but do not have an orthologue in C. reinhardtii, irrespective of their expression. Two approaches were employed.

First we identified thirteen candidate genes based on annotations of the transcripts and verified by reciprocal Blast searches as explained in material and methods (Table 5). Of these, in addition to the ACR3 family members discussed above, transcripts with following annotations were up-regulated: several isoforms of mitochondrial carrier protein MTM1 (comp10226_c0), which carries manganese for the mitochondrial superoxide dismutase, and of the MATE efflux family protein DETOXIFICATION 44 (comp12911_c0). To test for changes in gene expression caused by a low Cd exposure and metal specificity, qRT-PCR was performed on a selection of these candidate genes unique to $C$. acidophila (Table 4). Cells were collected for qRT-PCR at 1, 3 or $24 \mathrm{~h}$ after exposure to $\mathrm{Cd}(1 \mu \mathrm{M}), \mathrm{Cu}(1 \mathrm{mM}), \mathrm{Fe}(10 \mathrm{mM}), \mathrm{As}$ (III) (1mM) or As(V) (5 mM). Due to degraded cDNA Cd and Fe are represented by only two time points each.

Cd was noted to somewhat affect the expression of $18 \mathrm{~S}$ and therefore the relative mRNA expression levels of target genes were normalized against the levels of actin. Surprisingly, none of the tested transcripts were detected to be significantly induced by any of the added metals but significant down-regulation can be observed in most of them (Pair Wise Fixed Reallocation Randomisation test, $p<0.01$ ). These incongruences could be due to known methodological caveats in RNA-seq including different expression of the different isoforms, gene duplications or artifacts in assembly and annotation (Conesa et al. 2016). They demonstrate the importance of detailed functional studies of individual genes, although automated studies with massive input can offer useful information about general trends and serve as first step for further studies.

Secondly, we employed OrthoFinder to cluster genes and detect those unique to acidophiles (C. acidophila and Dunaliella acidophila). Eighteen genes present in both extremophilic species and at least one further algal 
species were extracted from the resulting orthologous groups (Table 6). Three of them (phytochelatin synthase CaPCS2, Arsenical-resistance protein ACR3 and multidrug efflux transporter AcrB) were detected both by the first method based on key word search for metal tolerance from the annotations and the second method based on filtering of orthologous groups.

Some of the key candidate genes highlighted in this study have been shown to enhance heavy metal tolerance in C. acidophila or other organisms. The phytochelatin synthase CaPCS2 was shown to be strongly induced by Cd in C. acidophila and cloning and expression of the gene in Escherichia coli clearly improved its cadmium resistance (Olsson et al. 2017). Cobalamin has been shown to protect against oxidative stress in the acidophilic iron-oxidizing bacterium Leptospirillum (Ferrer et al., 2016). Arsenical-resistance protein ACR3 is suggested to be a key trait to its arsenic tolerance in the arsenic hyperaccumulator Pteris vittata (Indriolo et al., 2010) and it might similarly enhance the tolerance to heavy metals in C. acidophila. Arsenite resistance efflux pump ArsB, which pumps arsenite and antimonite, but not arsenate or cadmium, was first described in E. coli (Wu et al., 1992. Our results suggest that these genes could be key traits for heavy-metal hypertolerance in C. acidophila. It has been proposed in other extremophiles as well that just a few key genes would be responsible for their hypertolerance to heavy-metals, for example Fer1 in the acidophilic archaeon Ferroplasma acidarmanus (BakerAustin et al., 2007).

\subsection{Phylogenetic distribution of candidate key genes involved in heavy-metal hyper-tolerance in C. acidophila} Most of the transcripts not present in C. reinhardtii with an annotation related to heavy metal tolerance are most closely related to genes in other green algae or vascular plants (Fig. 2A and Additional File 4). However, some transcripts get a first Blast hit in other algae, fungi, prokaryotes and amoebozoa. The phylogenetic distribution patterns in these genes can be explained by ancient gene duplications, loss in some lineages or 
horizontal gene transfer, and according to our results there are more than one explanation for the origin of these genes.

381

The mitochondrial carrier MTM1 (comp_10226, Fig. 2B), DETOXIFICATION 44 protein (comp_12911, Additional

File 4) and arsenite-antimonite efflux family (comp_15332, Additional File 4) include both green algae and

Chromalveolata among the most closely related genes. The phytochelatin synthase CaPCS2 (comp 11852,

Additional File 4), which is located within a clade of prokaryotic genes, has been functionally characterized and

biosynthesis protein CobW contains two gene copies in C. acidophila (comp15241_c0_seq3 and

comp15241_c0_seq6), of which one is similar to C. reinhardtii but the other is more similar to bacterial homologues (Fig. 2C).

Similarly, the genes not present in C. reinhardtii extracted with OrthoFinder have variable phylogenetic

distribution patterns (Fig. 3). Some of the genes are nested in a clade containing mainly bacteria (e.g. dioxygenase) and could be horizontally transferred. But for others, like transmembrane protein comp9629_c0_seq1 are closely related only to green algae and gene loss is a more likely explanation for their presence in C. acidophila but absence in C. reinhardtii.

\subsection{Codon code and aminoacid usage analysis}

The transcripts belonging to each of the analyzed species (Chlamydomonas acidophila, Chlamydomonas reinhardtii, Coccomyxa subellipsoidea, Dunaliella acidophila, Micromonas pusilla, Micromonas sp. RC299,

400 Ostreococcus lucimarinus and Volvox carteri) clearly clustered together with regards to their Relative Synonymous Codon Usage (Fig. 4a), showing the presence of distinct codon usage biases, even within 
403 (Fig. 4b). The majority of the transcripts clustered together regardless of their source organism, except for a

404 large set of transcripts from C. reinhardtii and the two Micromonas species, which clustered independently.

405 Both C. acidophila and D. acidophila showed similar utilization profiles for several aminoacids, particularly an

406 enrichment in serine and cysteine, and a depletion in glutamic and aspartic acids when compared to the non-

407 acidophilic species (Fig. 5a, Additional File 5). This depletion in Glu and Asp in acidophiles was also observed by

408 Goodarzi et al. (2008), but their study only included bacterial and archaeal genomes. To the best of our

409 knowledge, this is the first study which proposes that the same can also be true in eukaryotes. We further

410 calculated which GO-terms were significantly depleted in Glu and Asp, or enriched in Cys and Ser, in the

411 acidophilic species when compared to the non-acidophilic species (Fig. 5b, Additional File 6). In the four cases,

412 the significant GO-terms chiefly belonged to the binding, catalytic activity, and transporter activity base

413 categories. These four modifications (lower Glu, lower Asp, higher Cys and higher Ser contents) are likely

414 related to optimizations for acidic environments. For example, Glu and Asp are negatively charged in neutral

415 conditions, but become neutral at lower $\mathrm{pHs}$, which cancels their ability to stabilize proteins via salt bridges

416 (Anderson et al., 1990). On the other hand, the higher content of cystein could contribute to metal

417 detoxification and provide extra stability via disulfide bonds.

418

While the differences in total amino acid usage between organisms in acidophilic environments are usually caused mostly by a limited number of amino acids (Goodarzi et al. 2008), the observed differences in codon usage might be due to stronger selection pressure for codon optimization in extreme environments. Natural selection acting through external environmental factors can shape the genomic pattern of synonymous codon be true also in eukaryotes. 
426 The GC contents of a large amount of transcripts in all codon positions are different (Fig. 6) and can't be

427 explained by, for example, GC-content differences in a few horizontally transferred genes. Differences in the

428 first codon position affect the amino acid usage while differences in the third position are likely due to

preferences for different synonymous codons. Our results confirm that the overall genome-wide GC content is

the most significant parameter in explaining codon bias differences between organisms, suggested by Hershberg and Petrov (2008).

432

\subsection{Conclusions}

The results of this study, including the most complete published transcriptome of $C$. acidophila and a set of identified orthologous genes between eight green algae, increase the genomic information available on green algae and extremophilic eukaryotes, highlight the adaptations mechanisms used by algae to thrive in acidic environments, and provide a valuable resource for comparative studies on green algae from different habitats. Further work should focus on detailed analyses of individual genes and applied exploitation of the results, including engineering heavy metal tolerance in green algae for environmental and economic interests.

\section{ACKNOWLEDGEMENTS}

This work was supported by the Spanish Ministry of Economy and Competitivity (MINECO) [CGL2011-22540, AYA2011-24803]; the European Research Council (ERC) Advanced Grant [250350]. F. Puente-Sánchez was supported by the Spanish MINECO/FEDER [CTM2013-48292-C3-2-R]. We acknowledge the Data Intensive Academic Grid (DIAG) computing infrastructure (funded by National Science Foundation [0959894]) as well as CSC - Finnish IT Center for Science and the Finnish grid infrastructure (FGI) for the allocation of computational resources. Kimmo Mattila is acknowledged for help with setting up the OrthoFinder analysis pipeline. None of the co-authors declare a conflict of interest. 
Abe, J., Kubo, T., Takagi, Y., Saito, T., Miura, K., Fukuzawa, H., and Matsuda, Y. (2004) The transcriptional program of synchronous gametogenesis in Chlamydomonas reinhardtii. Curr Genet 46: 304-315.

Aguilera, A., and Amils, R., 2005. Tolerance to cadmium in Chlamydomonas sp. (Chlorophyta) strains isolated from an extreme acidic environment, the Tinto River (SW, Spain). Aquatic Toxicol 75: 316-329.

Aguilera, A., Manrubia, S.C., Gómez, F., Rodríguez, N., and Amils, R. (2006) Eukaryotic community distribution and its relationship to water physicochemical parameters in an extreme acidic environment, Rio Tinto (SW, Spain). Appl Environ Microbiol 72: 5325-5330.

Aguilera, A., Zettler, E., Gomez, F., Amaral-Zettler, L., Rodríguez, N., and Amils, R. (2007). Distribution and seasonal variability in the benthic eukaryotic community of Río Tinto (SW, Spain), an acidic, high metal extreme 462 environment. Syst Appl Microbiol 30: 531-546. Anderson, D E., Becktel, W.J., and Dahlquist, F.W. (1990). pH-induced denaturation of proteins: a single salt bridge contributes 3-5 kcal/mol to the free energy of folding of T4 lysozyme. Biochem 29: 2403-2408.

Altschul, S., Madden, T., Schaffer, A., Zhang, J., Zhang, Z., Miller, W., and Lipman D. (1997) Gapped BLAST and PSI-BLAST: a new generation of protein database search programs. Nucl Acid Res 25: 3389-3402. community structure across the tree of life in the extreme Río Tinto. ISME J 5: 42-50. arsenic resistance by the acidophilic archaeon 'Ferroplasma acidarmanus' Fer1. Extremophiles 11: 425-434.

471 Brayner, R., Dahoumane, S.A., Nguyen, J.N., Yéprémian, C., Djediat, C., Couté, A., and Fiévet, F. (2011) $1852-1858$. 
474 Buchheim, M.A., Turnel, M., Zimmer, E.A., and Chapman, R. (1990) Phylogeny of Chlamydomonas (Chlorophyta) 475 based on cladistic analysis of nuclear 18S rRNA sequence data. J Phycol 26: 689-699.

476 Capella-Gutiérrez, S., Silla-Martínez, J.M., and Gabaldón, T. (2009) trimAl: a tool for automated alignment

477 trimming in large-scale phylogenetic analyses. Bioinformatics 25: 1972-1973.

478 Castrillo, G., Sánchez-Bermejo, E., De Lorenzo, L., Crevillén, P., Fraile-Escaciano, A., T.C., M., et al. (2013) WRKY6 479 transcription factor restricts arsenate uptake and transposon activation in Arabidopsis. Plant Cell 25: 29444802957.

481 Cheng, X., Zhang, D., Cheng, Z., Keller, B., Ling, and H.-Q. (2009) A New Family of Ty1-copia-Like 482 retrotransposons originated in the tomato genome by a recent horizontal transfer event. Genetics 181: 11834831193.

484 Cicatelli, A., Todeschini, V., Lingua, G., Biondi, S., Torrigiani, P., and Castiglione, S. (2014) Epigenetic control of 485 heavy metal stress response in mycorrhizal versus non-mycorrhizal poplar plants. Environ Sci Pollut Res Int 21: $486 \quad 1723-37$.

487 Cobbett, C.S., and Goldsbrough, P. (2002) Phytochelatins and metallothioneins: roles in heavy metal 488 detoxification and homeostasis. Annu Rev Plant Physiol Plant Mol Biol 53: 159-182.

489 Conesa, A., Madrigal, P., Tarazona, S., Gomez-Cabrero, D., Cervera, A., McPherson, A., Wojciech Szcześniak, M., 490 Gaffney, D.J., Elo, L.L., Zhang, X., and Mortazavi, A. (2016) A survey of best practices for RNA-seq data analysis. 491 Genome Biol 17:13

492 De Wit, P., Pespeni, M.H., Ladner, J.T., Barshis, D.J., Seneca, F., Jaris, H., et al. (2012) The simple fool's guide to 493 population genomics via RNA-seq: an introduction to high-throughput sequencing data analysis. Mol Ecol 494 Resour 12: 1058-1067.

495 Díaz, S., Amaro, F., Rico, D., Campos, V., Benítez, L., Martín-González, A., et al. (2007) Tetrahymena 496 metallothioneins fall into two discrete subfamilies. PLoS ONE 2: e291 
Emms, D.M., and Kelly, S. (2015) OrthoFinder: solving fundamental biases in whole genome comparisons

498 dramatically improves orthologous group inference accuracy. Genome Biol 16: 157.

499 Fernández-Remolar, D.C., Rodríguez, N., Gómez, F., and Amils, R. (2003) Geological record of an acidic

500 environment driven by the iron hydrochemistry: the Tinto River system. J Geophys Res 108: 5080-5095.

501 Ferrer, A., Rivera, J., Zapata, C., Norambuena, J., Sandoval, Á., Chávez, R., Orellana, O., and Levicán, G. (2016)

502 Cobalamin protection against oxidative stress in the acidophilic iron-oxidizing bacterium Leptospirillum group II

503 CF-1. Front Microbiol 7: 748.

504 Flavell, A.J., Dunbar, E., Anderson, R., Pearce, S.R., Hartley, R., and Kumar, A. (1992) Ty1-copia group

505 retrotransposons are ubiquitous and heterogeneous in higher plants. Nucl Acids Res 20:3639-44.

506 García-Alcalde, F., Okonechnikov, K., Carbonell, J., Cruz, L.M., Götz, S., Tarazona, S., et al. (2012) Qualimap:

507 evaluating next generation sequencing alignment data. Bioinformatics 28: 2678-2679.

508 Ghamsari, L., Balaji, S., Shen, Y., Yang, X., Balcha, D., Fan, C., et al. (2011) Genome-wide functional annotation

509 and structural verification of metabolic ORFeome of Chlamydomonas reinhardtii. BMC Genomics, 12: S4.

510 Gómez-Álvarez V, Teal T.K., and Schmidt, T.M. (2009) Systematic artifacts in metagenomes from complex

511 microbial communities. ISME J 3:1314-1317.

512 González-Toril, E., Llobet-Brossa, E., Casamayor, E.O., Amann, R., and Amils, R. (2003) Microbial ecology of an

513 extreme acidic environment, the Tinto River. Appl Environ Microbiol 69: 4853-4865.

514 Goodarzi, H., Torabi, N., Najafabadi, H.S., and Archetti, M. (2008) Amino acid and codon usage profiles: adaptive

515 changes in the frequency of amino acids and codons. Gene 407: 30-41.

516 Grabherr, M.G., Haas, B.J., Yassour, M., Levin, J.Z., Thompson, D.A., Amit, I., et al. (2011) Full-length

517 transcriptome assembly from RNA-seq data without a reference genome. Nat Biotechnol 29:644-52.

518 Hanikenne, M., Motte, P., Wu, M.C.S., Wang, T., Loppes, R., and Matagne, R.F. (2005) A mitochondrial half-size

519 ABC transporter is involved in cadmium tolerance in Chlamydomonas reinhardtii. Plant Cell Environ 28: 863520873. 
521 Huelsenbeck, J.P., Ronquist, F., Nielsen, R., and Bollback, J.P. (2001) Bayesian inference of phylogeny and its 522 impact on evolutionary biology. Science 294: 2310-2314.

523 Hershberg, R., and Petrov, D.A. (2008) Selection on codon bias. Annu Rev Genet 42: 287-99.

524 Houot, L., Floutier, M., Marteyn, B., Michaut, M., Picciocchi, A., Legrain, P., et al. (2007) Cadmium triggers an 525 integrated reprogramming of the metabolism of Synechocystis PCC6803, under the control of the SIr1738 526 regulator. BMC Genomics 8: 350.

527 Hutchins, C.M., Simon, D.F., Zerges, W., Wilkinson, K.J. (2010) Transcriptomic signatures in Chlamydomonas 528 reinhardtii as Cd biomarkers in metal mixtures. Aquat Toxicol 100: 120-127.

529 Indriolo, E., Na, G., Ellis, D., Salt, D.E., and Banks, J.O. (2010) A Vacuolar arsenite transporter necessary for 530 arsenic tolerance in the arsenic hyperaccumulating fern Pteris vittata is missing in flowering plants. The Plant 531 Cell 6: 2045-2057.

532 Jamers, A., Blust, R., Coen, W.D., Griffin, J.L., and Jones O.A.H. (2013) An omics based assessment of cadmium 533 toxicity in the green alga Chlamydomonas reinhardtii. Aquat Toxicol 126: 355-364.

534 Katoh, K., Misawa, K., Kuma, K., and Miyata, T. (2002) MAFFT: a novel method for rapid multiple sequence 535 alignment based on fast Fourier transform. Nucleic Acid Res 30: 3059-3066.

536 Keller, M.D., Selvin, R.C., Claus, W., and Guillard, R.R.L. (1987) Media for the culture of oceanic 537 ultraphytoplankton. J Phycol 23: 633-638.

538 Lamai, C., Kruatrachue, M., Pokethitiyook, P., Upatham, E.S., and Soonthornsarathool, V. (2005) Toxicity and 539 accumulation of lead and cadmium in the filamentous green alga Cladophora fracta Kützing: a laboratory study. $540 \quad$ Science Asia 31: 121-127.

541 Langmead, B., Trapnell, C., Pop, M., and Salzberg, S.L. (2009) Ultrafast and memory-efficient alignment of short 542 DNA sequences to the human genome. Genome Biol 10: R25.

543 Larinov, A., Krause, A., Miller, W. (2005) Standard curve based method for relative real time PCR data 544 processing. BMC Bioinformatics 6: 62 
545 Leliaert, F., Smith, D.R., Moreau, H., Herron, M.D., Verbruggen, H., Delwiche, C.F., and De Clerck, O. (2012)

546 Phylogeny and molecular evolution of the green algae. Crit Rev Plant Sci 31: 1-46.

547 Li, H., Handsaker, B., Wysoker, A., Fennell, T., Ruan, J., Homer, N., et al., (2009) The Sequence alignment/map

548 (SAM) format and SAMtools. Bioinformatics 25: 2078-2079.

549 Li, B., and Dewey, C.N. (2011) RSEM: Accurate transcript quantification from RNA-Seq data with or without a 550 reference genome. BMC Bioinformatics 12: 323.

551 Lynn, D.J., Singer, G.A.C., and Hickey, D.A. (2002) Synonymous codon usage is subject to selection in

552 thermophilic bacteria. Nucl Acid Res 30: 4272-4277.

553 Manichaikul, A., Ghamsari, L., Hom, E.F., Lin, C., Murray, R.R., Chang, R.L., et al. (2009) Metabolic network

554 analysis integrated with transcript verification for sequenced genomes. Nat Methods 6: 589-592.

555 Marcais, G., and Kingsford, C. (2011). A fast, lock-free approach for efficient parallel counting of occurrences of

556 k-mers. Bioinformatics 27: 764-770.

557 Mclnerney, J.O. (1998) GCUA: general codon usage analysis. Bioinformatics 14: 372-373.

558 Merchant, S.S., Prochnik, S.E., Vallon, O., Harris, E.H., Karpowicz, S.J., Witman, G.B., et al. (2007) The

559 Chlamydomonas genome reveals the evolution of key animal and plant functions. Science 318: 245-250.

560 Miller, R., Wu, G., Deshpande, R. R., Vieler, A., Gärtner, K., Li, X., et al. (2010). Changes in transcript abundance

561 in Chlamydomonas reinhardtii following nitrogen deprivation predict diversion of metabolism. Plant Physiol

562 154: 1737-1752.

563 Müller, K., Quandt, D., Müller, J., and Neinhuis, C. (2005) PhyDE ${ }^{\circledR}$ : Phylogenetic Data Editor, version 0.995.

564 www.phyde.de

565 Nakada, T., Tomita, M., Wu, J.-T., and Nozaki, H. (2016) Taxonomic revision of Chlamydomonas subg.

566 Amphichloris (Volvocales, Chlorophyceae), with resurrection of the genus Dangeardinia and descriptions of

567 Ixipapillifera gen. nov. and Rhysamphichloris gen. nov. J Phycol 52: 283-304. 
568 Okamoto, O.K., Asano, C.S., Aidar, E., and Colepicolo, P. (1996) Effects of cadmium on growth and superoxide

569 dismutase activity of the marine microalga Tetraselmis gracilis (Prasinophyceae). J Phycol 32: 74-79.

570 Olsson, S., Puente-Sánchez, F., Gómez-Rodriguez, and M., Aguilera, A. (2015) Transcriptional response to copper

571 excess and identification of genes involved in heavy metal tolerance in the extremophilic microalga

572 Chlamydomonas acidophila. Extremophiles 19: 657-672.

573 Olsson, S., Penacho, V., Puente-Sánchez, F., Díaz, S., and Aguilera, A. (2017) Horizontal gene transfer of

574 phytochelatin synthases from bacteria to extremophilic green algae. Microbial Ecol 73: 50-60.

575 Puente-Sánchez, F., Olsson, S., and Aguilera, A. (2016). Comparative transcriptomic analysis of the response of

576 Dunaliella acidophila (Chlorophyta) to short-term cadmium and chronic natural metal-rich water exposures.

577 Microbial Ecol 72: 595-607.

578 Prasad, M.N.V., and Strzalka, K. (1999) Impact of heavy metals on photosynthesis. In: Heavy metal stress in

579 plants: from molecules to ecosystems. Prasad MNV, and Hagemeyer J, (eds). Berlin, Germany: Springer, pp.

$580 \quad 117-128$.

581 Rea, P.A., Vatamaniuk, O.K., and Rigden DJ. (2004) Weeds, Worms, and More. Papain's Long-Lost Cousin,

582 Phytochelatin Synthase. Plant Physiol 136: 2463-2474.

583 Robinson, M.D., McCarthy, D.J., and Smyth, G.K. (2010) EdgeR: a Bioconductor package for differential

584 expression analysis of digital gene expression data. Bioinformatics 26: 139-140.

Ronquist, F.,and Huelsenbeck, J.P. (2003) MRBAYES 3: Bayesian phylogenetic inference under mixed models.

586 Bioinformatics 19: 1572-1574.

587 Ronquist, F., Teslenko, M., van der Mark, P., Ayres, D., Darling, A., Höhna, S., et al. (2012) MrBayes 3.2: efficient

588 Bayesian phylogenetic inference and model choice across a large model space. Syst Biol 61: 539-542.

589 Rubinelli, P., Siripornadulsil, S., Gao-Rubinelli, F., and Sayre, R.T. (2002) Cadmium- and iron-stress-inducible gene 590 expression in the green alga Chlamydomonas reinhardtii: evidence for $\mathrm{H} 43$ protein function in iron assimilation.

591 Planta 215: 1-13. 
592 Schmieder, R., and Edwards, R. (2011) Quality control and preprocessing of metagenomic datasets.

593 Bioinformatics 27: 863-864.

594 Siaut, M., Cuiné, S., Cagnon, C., Fessler, B., Nguyen, M., Carrier, P., et al. (2011) Oil accumulation in the model 595 green alga Chlamydomonas reinhardtii: characterization, variability between common laboratory strains and 596 relationship with starch reserves. BMC Biotechnol 11: 7.

597 Stamatakis, A. (2006) RAxML-VI-HPC: Maximum likelihood-based phylogenetic analyses with thousands of taxa 598 and mixed models. Bioinformatics 22: 2688-2690.

599 Stamatakis, A., Hoover, P., and Rougemont, J. (2008) A rapid bootstrap algorithm for the RAxML Web servers. 600 Syst Biol 57: 758-771.

601 Stöver BC, and Müller KF. (2010) TreeGraph 2: Combining and visualizing evidence from different phylogenetic 602 analyses. BMC Bioinformatics 11: 7.

603 Wang, L., Yang, F., Chen, H., Fan, Z., Zhou, Y., Lu, J., and Zheng, Y. (2016) Antimicrobial cocktails to control 604 bacterial and fungal contamination in Chlamydomonas reinhardtii cultures. Biotechniques 60: 145-149. 605 Wang, S., Zhang, D., and Pan, X. (2013) Effects of cadmium on the activities of photosystems of Chlorella 606 pyrenoidosa and the protective role of cyclic electron flow. Chemosphere 93: 230-237.

607 Wit Wu, J., Tisa, L.S., and Rosen, B.P. (1992) Membrane topology of the ArsB protein, the membrane subunit of 608 an anion-translocating ATPase. J Biol Chem 267: 12570-12576.

609 Zeldovich, K.B., Berezovsky, I.N., and Shakhnovich, E.I. (2007) Protein and DNA Sequence Determinants of 610 Thermophilic Adaptation. PLoS Comput Biol 3: e5.

611 Zhang, W., Tana, N., and Li, S.F. (2014) NMR-based metabolomics and LC-MS/MS quantification reveal metal612 specific tolerance and redox homeostasis in Chlorella vulgaris. Mol Biosyst 10: 149-160. 
FIGURE CAPTIONS

Figure 1. Phylogenetic relationships based on 488 nuclear single orthologous genes clustered with OrthoFinder and present in all eight species (Chlamydomonas acidophila, Dunaliella acidophila, Chlamydomonas reinhardtii, Coccomyxa subellipsoidea, Micromonas pusilla, Micromonas sp. RCC299, Osterococcus lucimarinus and Volvox carteri). The trees represent the majority consensus of trees sampled after stationarity in the Bayesian analysis. Posterior probability values from the Bayesian inference are indicated above, the corresponding bootstrap values of the maximum likelihood analysis below the branches.

Figure 2. Simplified phylogenetic analyses of transcripts coding for genes with an annotation related to heavy metal tolerance and present in C. acidophila but not in C. reinhardtii. The phylograms represent the majority consensus of trees sampled after stationarity in the Bayesian analysis. PP values equal or greater than 0.50 are shown above branches. The scale bar indicates relative distance between different sequences based on mutation rate. A) peroxisome isogenesis protein comp_10128 B) mitochondrial carrier comp 10226 C) cobalamin biosynthesis protein CobW comp_15241.

Figure 3. Simplified phylogenetic analyses of transcripts coding for genes that are involved in heavy metal tolerance and are present in C. acidophila but not in C. reinhardtii extracted from the results from the search for orthologous genes with OrthoFinder. The phylograms represent the majority consensus of trees sampled after stationarity in the Bayesian analysis. PP values equal or greater than 0.50 are shown above branches. The scale bar indicates relative distance between different sequences based on mutation rate. A) 2-hydroxyacyl-CoA lyase comp18202_c0_seq7 B) Dioxygenase comp13804_c0_seq4 C) Transmembrane protein 230 comp9629_c0_seq1 D) Cocaine esterase comp3348_c0_seq1 E) SDR-family protein with acetoacetyl-CoA reductase activity comp_14433_c0_seq1. 
Figure 4. a) Correspondence analysis showing the distribution of transcripts (points) according to their Relative Synonymous Codon Usage distribution and b) Correspondence analysis showing the distribution of transcripts (points) according to the aminoacid usage bias of their predicted ORFs. The percentage of inertia explained by each axis is indicated in the axis caption. Transcripts are coloured by their source genome.

Figure 5. a) Distribution of Glu, Asp, Cys and Ser contents in the predicted ORFs from the eight species analyzed in this study. The ORFs included in the green area (low Glu, low Asp, high Cys and high Ser) were subjected to a GO-term enrichment analysis between the two acidophilic species and the rest. b) Summary of the Molecular Function GO-terms found to be significantly enriched $(p<0.05)$ in acidophiles versus non-acidophiles, when focusing in the low Glu, low Asp, high Cys, and high Ser fractions of the proteomes. Full results are provided in Additional File 6.

Figure 6. Scatterplots showing the GC content on each transcript (points) in the three different codon positions. Transcripts are coloured by their source genome. 


\section{TABLES WITH CAPTIONS}

Table 1. Quantitative real-time RT-PCR standard-curve parameters for selected transcripts present in C.

acidophila but not in C. reinhardtii and the expression control (housekeeping) genes $18 \mathrm{~S}$ rNA and actin. S= slope, $\mathrm{R}^{2}=$ correlation coefficient, $\mathrm{E}=$ amplification efficiency.

\begin{tabular}{|l|l|l|l|}
\hline Gene & S & R2 & E \\
\hline ACR3 & -2.858 & 0.99 & 2.24 \\
\hline Arsenite transporter & -2.885 & 0.97 & 2.22 \\
\hline AcrB & -3.04 & 0.99 & 2.13 \\
\hline $\begin{array}{l}\text { Glutathione-regulated } \\
\text { potassium-efflux family }\end{array}$ & -2.823 & 1 & 2.26 \\
\hline MATE efflux protein & -3.079 & 0.99 & 2.11 \\
\hline $\begin{array}{l}\text { Arsenite-antimonite efflux } \\
\text { family }\end{array}$ & -2.96 & 0.96 & 2.18 \\
\hline
\end{tabular}

Table 2. Primers used for quantitative real-time RT-PCR used in this study. For each region, forward (F) and reverse

(R) primers are indicated, as well as product size.

\begin{tabular}{|c|c|c|c|c|}
\hline Gene & Primer name & 5' Sequence 3' & $F / R$ & Product size (bp) \\
\hline \multirow{2}{*}{$\begin{array}{l}\text { Multidrug efflux } \\
\text { transporter AcrB } \\
\text { comp_16471 }\end{array}$} & comp16471_AcrB-F & GTAGGCATTCCCTTGCTGTC & $\mathrm{F}$ & \multirow{2}{*}{89} \\
\hline & comp16471_AcrB-R & CCAAGGACCAAAACAAGCAT & $\mathrm{R}$ & \\
\hline \multirow{2}{*}{ ACR3 comp_14907 } & comp14907_ACR3-F & ACTTTTGGCTTCTGGGAGGT & $\mathrm{F}$ & \multirow{2}{*}{106} \\
\hline & comp14907_ACR3-R & TTTCACCATAAGCCCAGACC & $\mathrm{R}$ & \\
\hline \multirow{2}{*}{$\begin{array}{l}\text { Arsenite transporter } \\
\text { comp_15936 }\end{array}$} & comp15936_ArsB-F & AATGTTACGGCAAAGCGAAC & $\mathrm{F}$ & \multirow{2}{*}{100} \\
\hline & comp15936_ArsB-R & CAGTCACTGGCGAGCTCATA & $\mathrm{R}$ & \\
\hline \multirow{2}{*}{$\begin{array}{l}\text { MATE efflux protein } \\
\text { comp_12911 }\end{array}$} & comp12911_MATE-F & ACTTTGGGTTCATGGCTTTG & $\mathrm{F}$ & \multirow{2}{*}{98} \\
\hline & comp12911_MATE-R & САСТCCTGCCAGTCCTAACC & $\mathrm{R}$ & \\
\hline \multirow{2}{*}{$\begin{array}{l}\text { Arsenite-antimonite } \\
\text { efflux family } \\
\text { comp_15332 }\end{array}$} & comp15332_MATE-F & CTAACACTCCTGTGGCAGCA & $\mathrm{F}$ & \multirow{2}{*}{125} \\
\hline & comp15332_MATE-R & CAGCCTGTTAAGCCCTTTTG & $\mathrm{R}$ & \\
\hline \multirow{2}{*}{$\begin{array}{l}\text { Glutathione-regulated } \\
\text { potassium-efflux family } \\
\text { comp_16013 }\end{array}$} & comp16013_K-efflux-F & CGCTAGAAATTCCCAACCAG & $\mathrm{F}$ & \multirow{2}{*}{87} \\
\hline & comp16013_K-efflux-R & GCATTTCTTTGGACCTCCAT & $\mathrm{R}$ & \\
\hline
\end{tabular}


Table 3. Sequence statistics on A) Illumina sequencing and for comparison, statistics on 454 reads from Olsson et al., (2015) are also shown. B) transcriptome assembly with Trinity using a hybrid assembly strategy combining 454 reads with Illumina reads.

\begin{tabular}{|c|c|c|c|c|c|}
\hline \multicolumn{6}{|c|}{ Illumina sequencing } \\
\hline Library & Condition & Raw reads & Input bases (Gb) & Trimmed reads & Discarded sequences (including duplicates) \\
\hline $\mathrm{J} 1$ & Reinhardtii-Oh & 21243002 & 1.61 & 451530 & 9504620 \\
\hline $\mathrm{J} 2$ & Reinhadtii-3h & 21819884 & 1.66 & 459756 & 10645037 \\
\hline $\mathrm{J3}$ & Reinhadtii-6h & 19474228 & 1.48 & 438115 & 9709139 \\
\hline J5 & Acidophila-3h & 22928585 & 1.74 & 503621 & 11287054 \\
\hline J6 & Acidophila-6h & 22006149 & 1.67 & 466332 & 11948336 \\
\hline \multicolumn{6}{|c|}{454 data from Olsson et al., (2015) } \\
\hline \multicolumn{2}{|c|}{ Input 454 reads } & \multicolumn{2}{|c|}{ Simulated Illumina reads } & \multicolumn{2}{|c|}{ Normalized simulated pairs } \\
\hline
\end{tabular}

B)

\begin{tabular}{|l|l|}
\hline \multicolumn{2}{|l|}{ Hybrid assembly } \\
\hline Input pairs & 7717263 \\
\hline Input SE reads & 33998990 \\
\hline Bases in assembly (Mb) & 293 \\
\hline Trinity genes & 47411 \\
\hline Trinity isoforms & 151449 \\
\hline Isoform median length & 1398 \\
\hline Isoform mean length & 1936.66 \\
\hline Range of isoform lengths & $201-19360$ \\
\hline Isoform N50 & 3212 \\
\hline Isoform mean coverage & $54.62 X$ \\
\hline Isoform std coverage & $107.15 X$ \\
\hline Isoforms after filtering & 129188 \\
\hline $\begin{array}{l}\text { Isoforms after filtering with } \\
\text { nr BLAST matches }\end{array}$ & 87676 \\
\hline
\end{tabular}

Table 4. Results of gene expression analysis by qRT-PCR of selected genes present in C. acidophila but not in $C$. reinhardtii. The cells were collected at 1,3 or 24 hours after exposure to $1 \mu \mathrm{M} \mathrm{Cd}$ solution $\left(\mathrm{CdCl}_{2} \times 2 \frac{1}{2} \mathrm{H}_{2} \mathrm{O}\right)$, $1 \mathrm{mM} \mathrm{Cu}\left(\mathrm{CuSO}_{4} \times 5 \mathrm{H}_{2} \mathrm{O}\right), 10 \mathrm{mM} \mathrm{Fe}\left(\mathrm{FeSO}_{4} \times 7 \mathrm{H} 2 \mathrm{O}\right), 1 \mathrm{mM}$ As (III) $\left(\mathrm{AsNaO}_{2}\right)$ or $5 \mathrm{mM} \mathrm{As}(\mathrm{V})\left(\mathrm{Na}_{2} \mathrm{HAsO}_{4}\right)$. The relative 
mRNA expression levels of target genes were normalized against the levels of actin and $18 \mathrm{~S}$. The fold induction and SD for each target gene is shown. ACR3 comp14907 = arsenical-resistance protein ACR3, ACR3 comp15936 = ACR3 family arsenite transporter, MATE comp12911 = MATE efflux protein, Arsenite-antimonite comp15332 = Arsenite-antimonite efflux family, AcrB comp16471 = Multidrug efflux transporter AcrB, MATE comp12911 = MATE efflux protein. $\mathrm{Nd}=$ Not defined.

\begin{tabular}{|l|l|l|l|l|l|l|}
\hline & $\begin{array}{l}\text { ACR3 } \\
\text { comp14907 }\end{array}$ & $\begin{array}{l}\text { ACR3 } \\
\text { comp15936 }\end{array}$ & $\begin{array}{l}\text { MATE } \\
\text { comp12911 }\end{array}$ & $\begin{array}{l}\text { Arsenite- } \\
\text { antimonite } \\
\text { comp15332 }\end{array}$ & $\begin{array}{l}\text { AcrB } \\
\text { comp1647 } \\
\mathbf{1}\end{array}$ & $\begin{array}{l}\text { MATE } \\
\text { comp12911 }\end{array}$ \\
\hline Cd 1h & $-12,98 \pm 1,97$ & $-513 \pm 126,1$ & $-675,6 \pm 79,1$ & $-2327 \pm 574$ & $-761 \pm 112$ & $-8,1 \pm 2,5$ \\
\hline Cd 24h & $-6,36 \pm 0,8$ & $-14,3 \pm 3,6$ & $-20,62 \pm 3,0$ & $-2862 \pm 599$ & $-609 \pm 113$ & $-12,5 \pm 3,3$ \\
\hline As(III) 1h & $0,52 \pm 0,06$ & $-1,19 \pm 0,09$ & $-1,04 \pm 0,3$ & $-10,2 \pm 1,4$ & $-13,5 \pm 2$ & $-0,7 \pm 0,1$ \\
\hline As(III) 3h & $-293,79 \pm 36,59$ & $-1824 \pm 138$ & $-731,7 \pm 135,4$ & $-15158 \pm 1135$ & $-3142 \pm 378$ & $-4,2 \pm 1,2$ \\
\hline As(III) 24h & $-11,7 \pm 1,45$ & $-3445 \pm 261$ & $-314,9 \pm 46,1$ & $-3531 \pm 864$ & $-476 \pm 70$ & $-18 \pm 5,4$ \\
\hline Cu 1h & $4,15 \pm 0,52$ & $-3687 \pm 1012$ & $3,38 \pm 1,1$ & $-3,8 \pm 1,2$ & $-6639 \pm 786$ & $-55 \pm 11,7$ \\
\hline Cu 3h & $4,65 \pm 0,5$ & $-6358 \pm 2424$ & $5,6 \pm 0,4$ & $-11,48 \pm 3,3$ & $-690 \pm 81$ & $-1,5 \pm 0,4$ \\
\hline Cu 24h & $1,04 \pm 0,12$ & $-170,2 \pm 35,7$ & $-26,02 \pm 3,9$ & $-554,9 \pm 89$ & $-207 \pm 30$ & $-47 \pm 12$ \\
\hline Fe 3h & $\mathrm{Nd}$ & $\mathrm{Nd}$ & $1,31 \pm 0,2$ & $-819,8 \pm 94,7$ & $\mathrm{Nd}$ & $\mathrm{Nd}$ \\
\hline Fe 24h & $0,36 \pm 0,05$ & $1,18 \pm 0,2$ & $0,8 \pm 0,1$ & $-2,2 \pm 0,3$ & $\mathrm{Nd}$ & $\mathrm{Nd}$ \\
\hline
\end{tabular}


Table 5. Transcripts coding for genes that are involved in heavy metal tolerance present in C. acidophila but not in C. reinhardtii based on transcript annotations.

\begin{tabular}{|c|c|c|c|c|}
\hline Contig name & Putative function & BLAST top match organism & $\begin{array}{l}\text { BLAST match } \\
\text { accession }\end{array}$ & E-value \\
\hline comp10128_c0_seq1 & Peroxisome isogenesis & Coccomyxa subellipsoidea & XP_005647114 & $3.01 E-39$ \\
\hline comp10226_c0_seq5 & Mitochondrial carrier & Coccomyxa subellipsoidea & XP_005652123 & $3.95 \mathrm{E}-27$ \\
\hline comp11852_c0_seq1 & Phytochelatin synthase & Calothrix sp. & YP_007140091 & $6.44 \mathrm{E}-30$ \\
\hline comp13602_c0_seq11 & NRAMP family protein & Volvox carteri & XP_002947173 & $5.89 \mathrm{E}-153$ \\
\hline comp14042_c0_seq2 & ABC-ATPase & Coccomyxa subellipsoidea & XP_005643834 & $1.62 \mathrm{E}-92$ \\
\hline comp14907_c0_seq53 & Arsenical-resistance protein ACR3 & Coccomyxa subellipsoidea & XP_005649016 & $1.99 \mathrm{E}-29$ \\
\hline comp15241_c0_seq3 & Cobalamin biosynthesis CobW & Chlamydomonas reinhardtii & XP_001699037 & $7.26 \mathrm{E}-60$ \\
\hline comp16013_c0_seq1 & $\begin{array}{l}\text { Glutathione-regulated potassium- } \\
\text { efflux system }\end{array}$ & Volvox carteri & XP_002953483 & $8.63 \mathrm{E}-37$ \\
\hline comp16471_c0_seq3 & Multidrug efflux transporter AcrB & Zea mays & AFW59203 & $4.15 E-58$ \\
\hline comp17557_c1_seq9 & $\begin{array}{l}\text { Multidrug resistance-associated } \\
\text { protein }\end{array}$ & Coccomyxa subellipsoidea & XP_005651467 & $1.59 \mathrm{E}-144$ \\
\hline
\end{tabular}


Table 6. Transcripts coding for genes that are involved in heavy metal tolerance present in C. acidophila but not in C. reinhardtii filtered from orthologous groups defined with OrthoFinder. Orthologous groups with annotations related to heavy metal tolerance and detoxification are marked with *. In the case of groups including several transcripts, the Blast hit organism and accession refers to the first one.

\begin{tabular}{|c|c|c|c|c|c|}
\hline $\begin{array}{l}\text { Orthologous } \\
\text { group }\end{array}$ & Contig name & Putative function & $\begin{array}{l}\text { BLAST top match } \\
\text { organism }\end{array}$ & $\begin{array}{l}\text { BLAST match } \\
\text { accession }\end{array}$ & E-value \\
\hline OG0001276 & comp13064_c0_seq2, comp15004_c0_seq1 & Tripeptidyl-peptidase 1 & $\begin{array}{l}\text { Polysphondylium } \\
\text { pallidum }\end{array}$ & EFA84081 & $1.38 \mathrm{e}-17$ \\
\hline OG0001752 & comp12567_c0_seq1 & Amino acid permease 2 & Capsella rubella & EOA20485 & $3.33 e-60$ \\
\hline OG0001782 & comp15790_c0_seq1 & $\begin{array}{l}\text { Alpha-1,3- } \\
\text { glucosyltransferase }\end{array}$ & $\begin{array}{l}\text { Coccomyxa } \\
\text { subellipsoidea }\end{array}$ & XP_005651392 & $9.52 \mathrm{e}-92$ \\
\hline OG0003420 & comp16380_c0_seq1 & $\begin{array}{l}\text { Metal-nicotianamine } \\
\text { transporter }\end{array}$ & Amborella trichopoda & ERN09450 & $5.88 \mathrm{e}-32$ \\
\hline OG0003495 & comp18202_c0_seq7 & 2-hydroxyacyl-CoA lyase & Galdieria sulphuraria & XP_005708092 & 0.0 \\
\hline OG0004374 & comp18062_c0_seq1 & $\begin{array}{l}\text { Abhydrolase domain- } \\
\text { containing protein }\end{array}$ & $\begin{array}{l}\text { Dictyostelium } \\
\text { purpureum }\end{array}$ & XP_002957250 & $2.33 e-05$ \\
\hline OG0005487* & comp14907_c0_seq15 & $\begin{array}{l}\text { Arsenical-resistance } \\
\text { protein ACR3 }\end{array}$ & $\begin{array}{l}\text { Coccomyxa } \\
\text { subellipsoidea }\end{array}$ & XP_005649016 & $1.45 \mathrm{e}-85$ \\
\hline OG0005928* & comp13804_c0_seq4 & dioxygenase & Volvox carteri & XP_002957190 & $2.32 \mathrm{e}-53$ \\
\hline OG0006489 & comp13735_c0_seq3 & Snurportin-1 & Physcomitrella patens & XP_001763666 & $5.34 \mathrm{e}-49$ \\
\hline OG0006590* & comp16471_c0_seq1 & $\begin{array}{l}\text { multidrug efflux } \\
\text { transporter AcrB }\end{array}$ & Arabidopsis thaliana & OAP00250 & $5.35 e-63$ \\
\hline OG0007003 & comp14473_c0_seq1 & Ankyrin-1 & Aegilops tauschii & EMT31987 & $3.47 e-56$ \\
\hline OG0007890* & comp9629_c0_seq1 & $\begin{array}{l}\text { Transmembrane protein } \\
230\end{array}$ & Physcomitrella patens & XP_001772694 & $2.89 \mathrm{e}-17$ \\
\hline OG0010052 & comp17871_c0_seq9 & Hisitidine kinase & Synechocystis sp. & WP_009631601 & $1.298 \mathrm{e}-39$ \\
\hline
\end{tabular}


SUPPLEMENTARY MATERIAL

Additional File 1. C. reinhardtii transcripts with a log2 fold change higher than 6 and FPKM of more than 20 in at least one sample. Normalized expression of the transcripts in each condition ( $0 \mathrm{~h}, 3 \mathrm{~h}$ and $6 \mathrm{~h}$ after exposure to cadmium), their putative annotation and sequence length are shown. Transcripts are ordered by FPKM values at $6 \mathrm{~h}$.

Additional File 2. C. acidophila RT46 transcripts with a log2 fold change higher than 6 and FPKM of more than 20 in at least one sample. Normalized expression of the transcripts in each condition (0h, 3h and $6 \mathrm{~h}$ after exposure to cadmium) and their putative annotation are shown. Transcripts are ordered by FPKM values at $6 \mathrm{~h}$.

Additional File 3. Data matrix used for the phylogenomic analysis, containing 488 single orthologous groups of proteins clustered with OrthoFinder present in all species (Chlamydomonas acidophila, Chlamydomonas reinhardtii, Coccomyxa subellipsoidea, Dunaliella acidophila, Micromonas pusilla, Micromonas sp. RC299, Osterosoccus lucimarinus and Volvox carteri).

Additional File 4. Simplified phylogenetic analyses of transcripts coding for genes with an annotation related to heavy metal tolerance and present in C. acidophila but not in C. reinhardtii. The trees represent the majority consensus of trees sampled after stationarity in the Bayesian analysis. PP values equal or greater than 0.50 are shown above branches. A) ACR3 comp_14907 and comp_15936 B) DETOXIFICATION 44 protein comp_12911 C) NRAMP family comp_13602 D) ABC-ATPase comp_14042 E) Arsenite-antimonite efflux family comp_15332 F) Glutathione-regulated potassium-efflux comp_16013 G) Multidrug efflux transporter AcrB comp_16471 H) Multidrug resistance-associated protein comp_17557. 
Additional File 5. Aminoacid utilization profiles for the different species. There is one cumulative frequency plot for each aminoacid.

Additional File 6. GO terms enriched in acidophiles versus non-acidophiles in the low-Glu, low-Asp, high-Cys, and high-Ser fractions of the proteome. 
Micromonas pusilla

- Micromonas strain RCC299

\section{Osterococcus lucimarinus}

Coccomyxa subellipsoidea

100

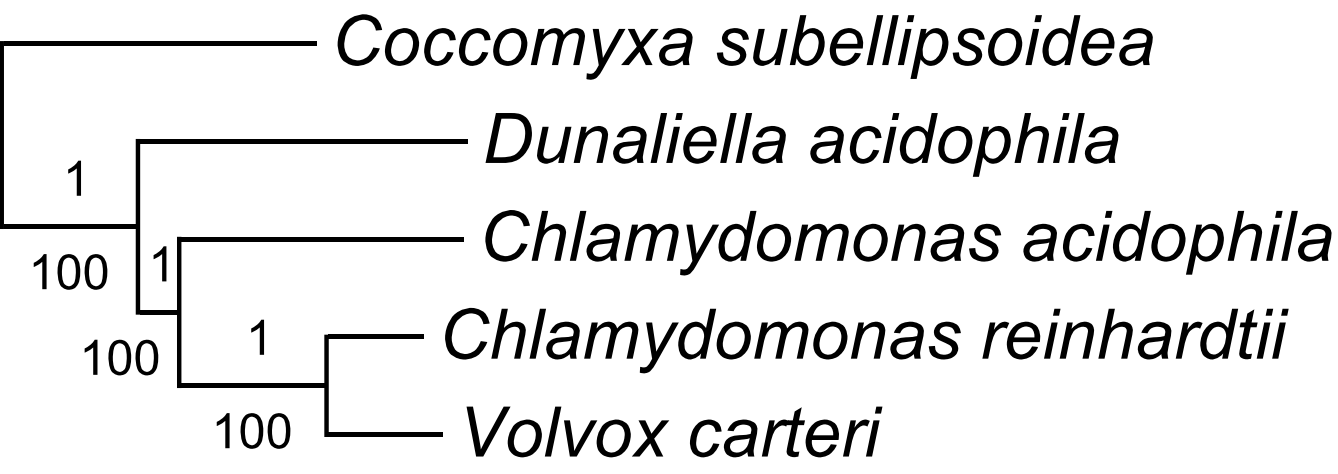




\section{Fungi}

Fungi

Fungi

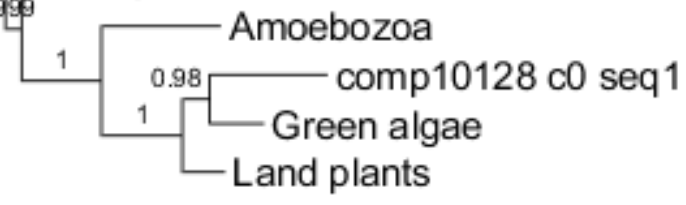

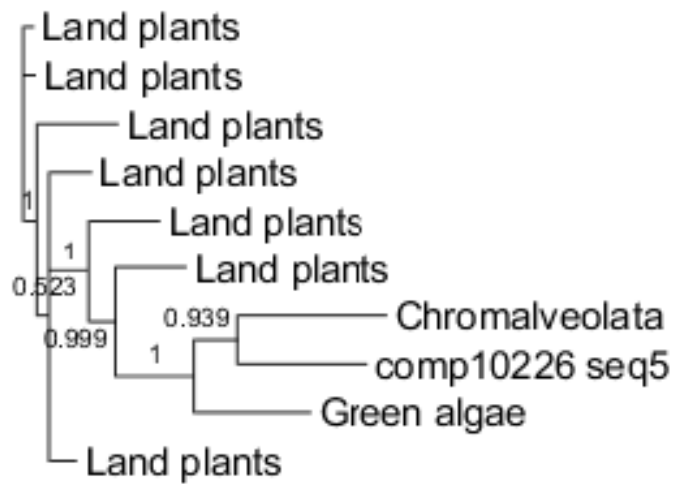

B

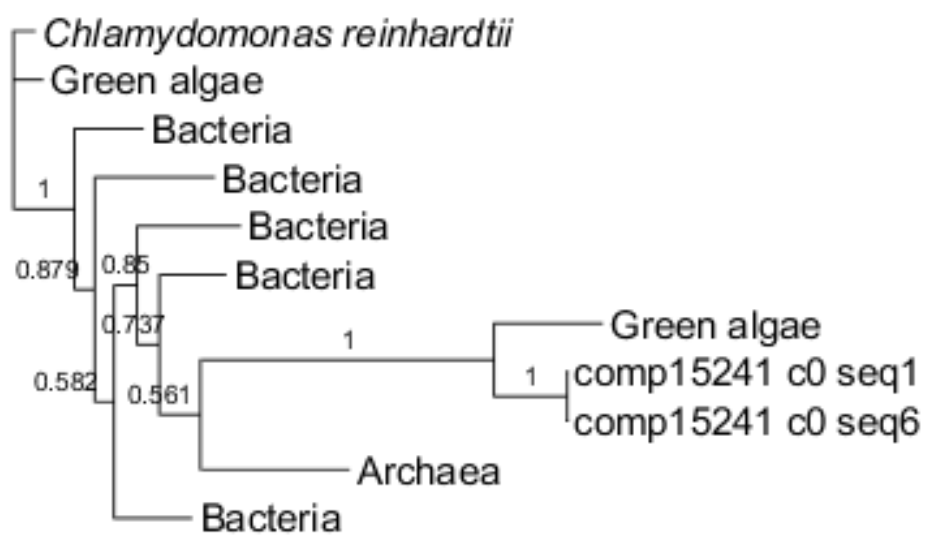




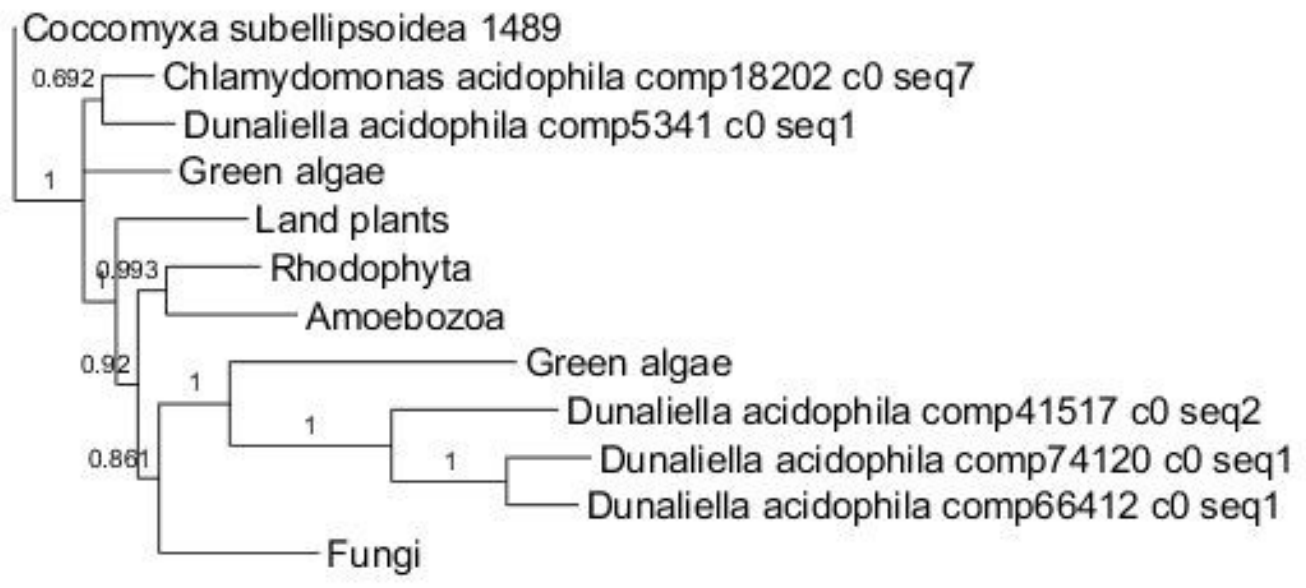

A) OG0003495: 2-hydroxyacyl-CoA lyase comp18202_c0_seq7

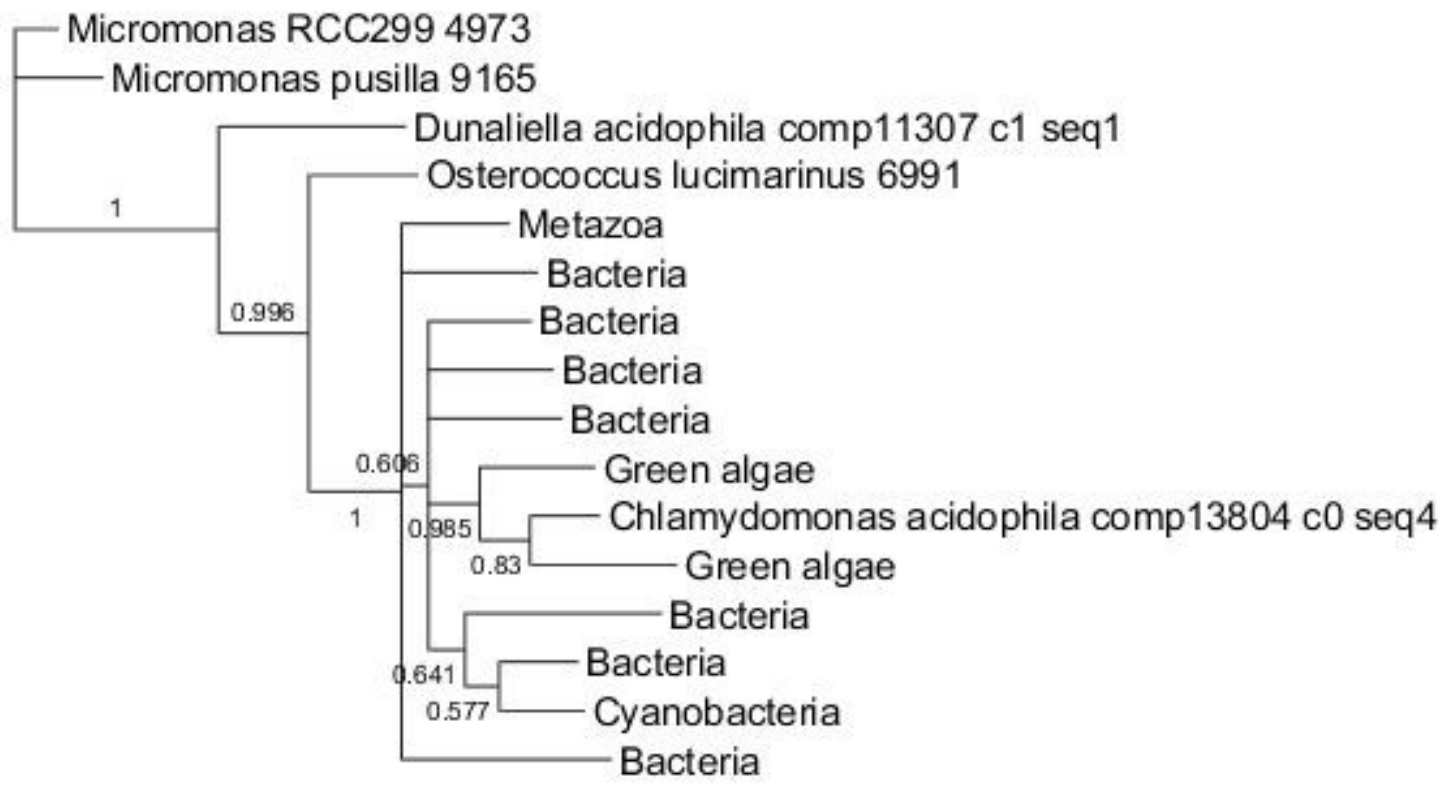

B) OG0005928: Dioxygenase comp13804_c0_seq4

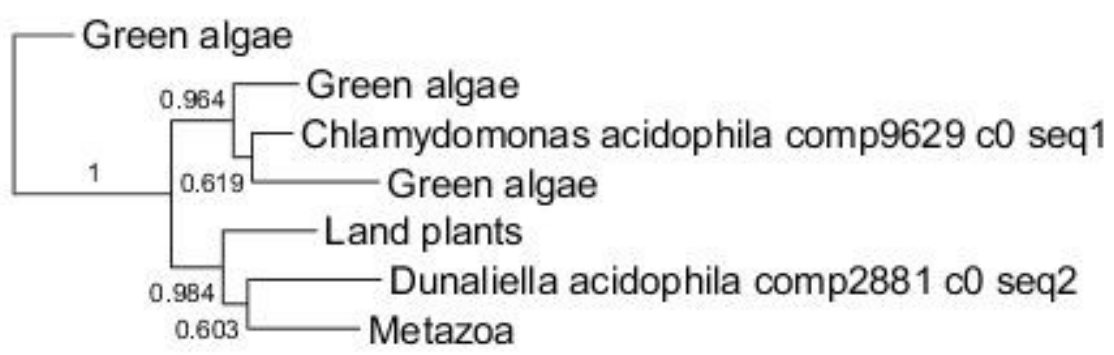

C) OG0007890: Transmembrane protein 230 comp9629_c0_seq1 
- Green algae

0.999 Dunaliella acidophila comp 136116 c0 seq1 - Green algae

1 Chlamydomonas acidophila comp3348 c0 seq1 Bacteria

D) OG0008459: Cocaine esterase comp3348_c0_seq1

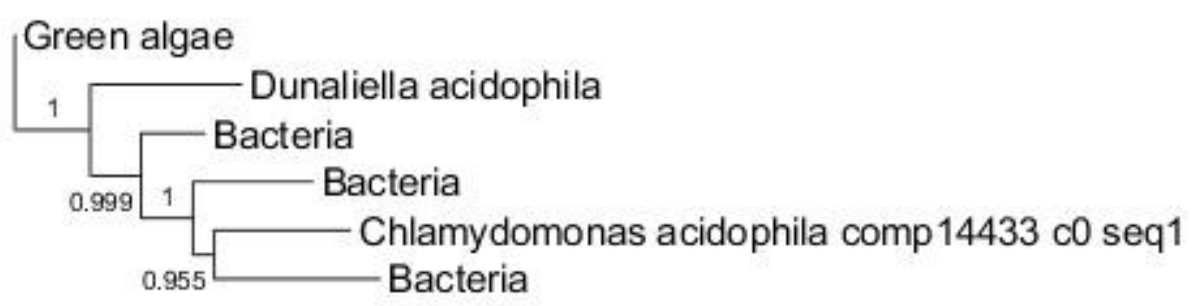

E) OG0009876: SDR-family protein with acetoacetyl-CoA reductase activity comp_14433_c0_seq1 

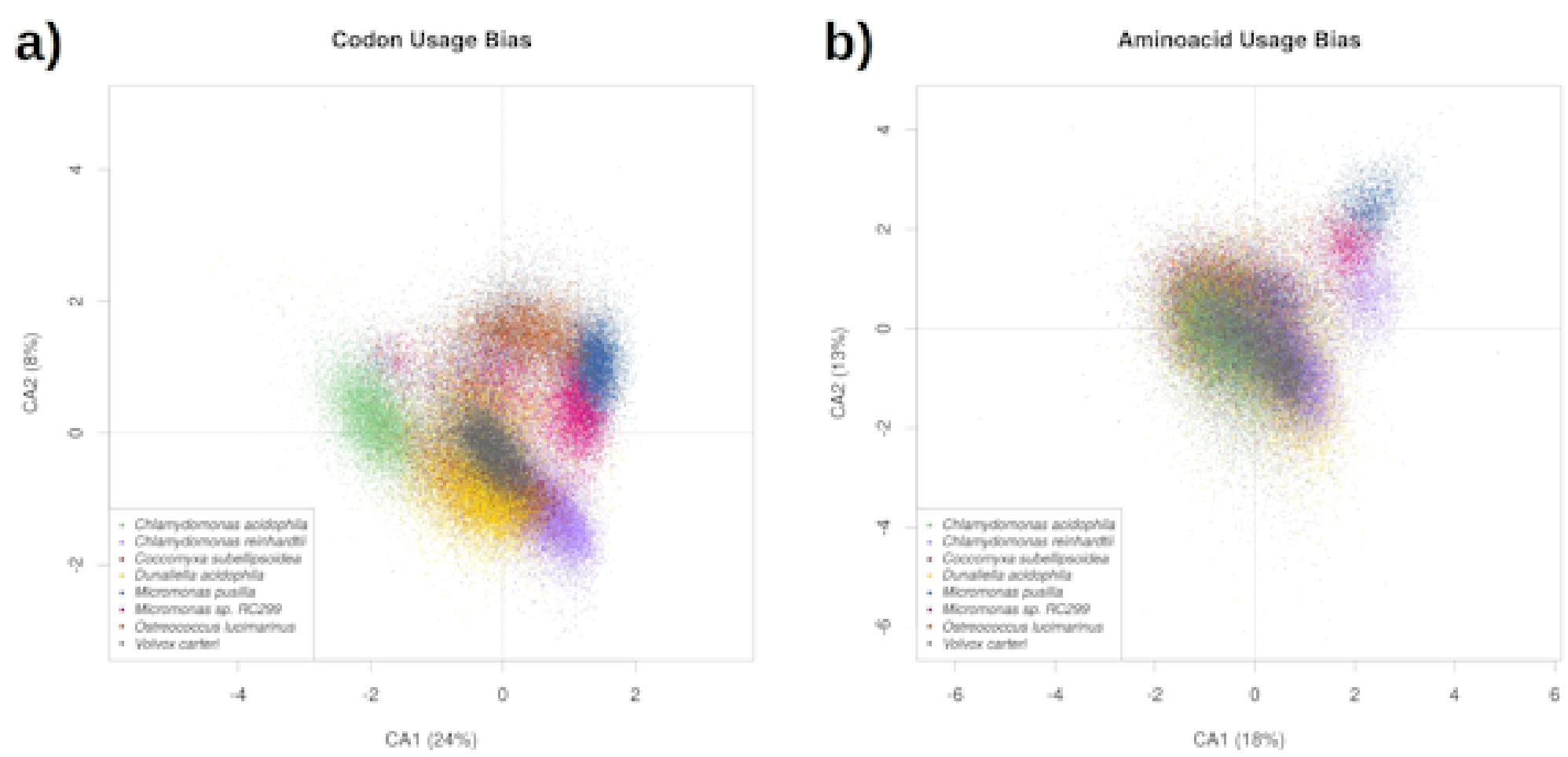


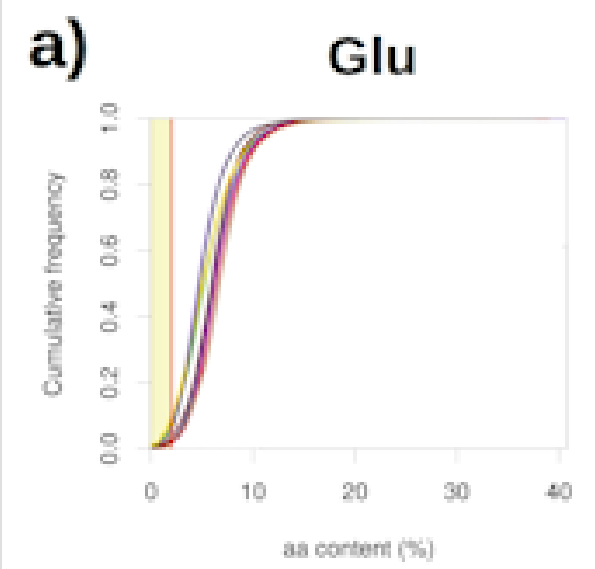

Cys

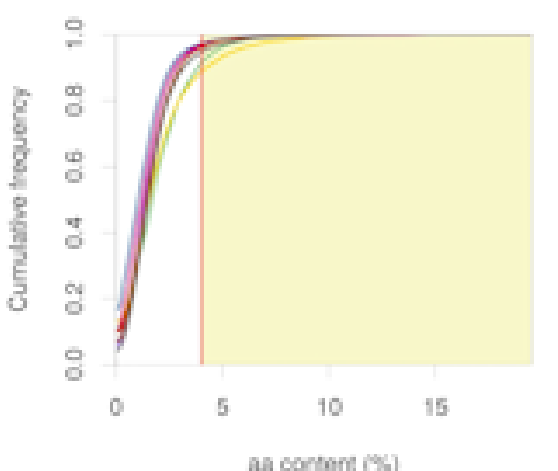

aa cortent (\%)

- Chlamydomonas acidophila

- Chlamydomonas reinhardill

- Coccomyxa subellipsoidea

- Dunaliella acidophila

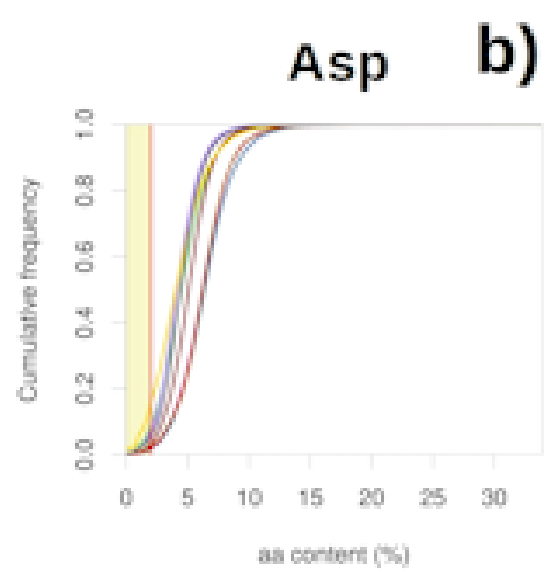

Glu-depleted GOterms in acidophiles

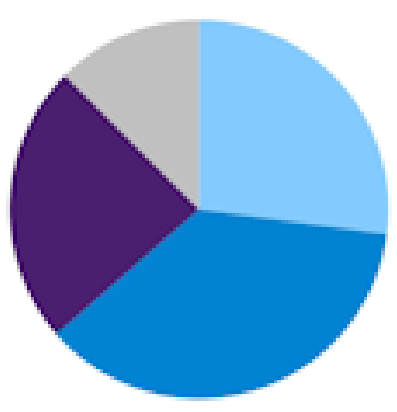

Ser

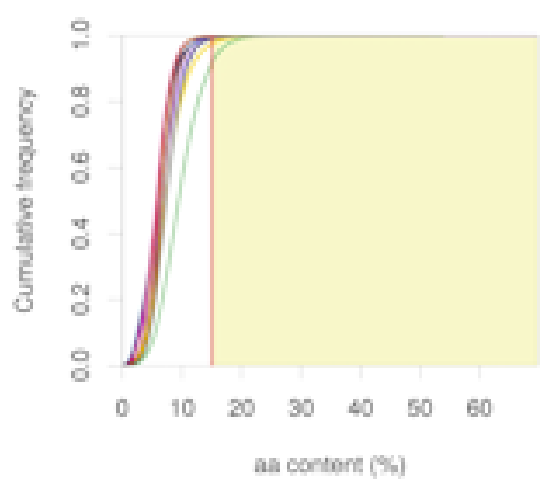

- Micromonas pusilla

- Micromonas sp. RC299

- Ostreococcus hucimarinus

- Volvox carteri
Cys-enriched GOterms in acidophiles

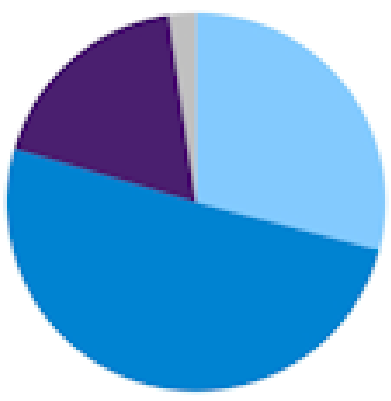

= binding

catalytic activity
Asp-depleted GOterms in acidophiles

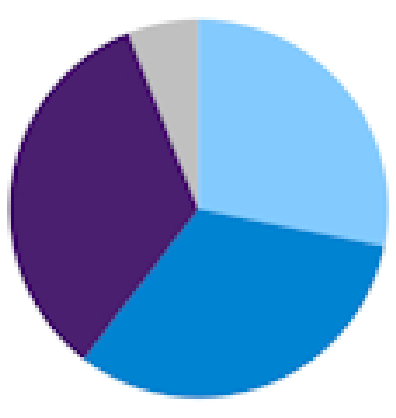

Ser-enriched GOterms in acidophiles

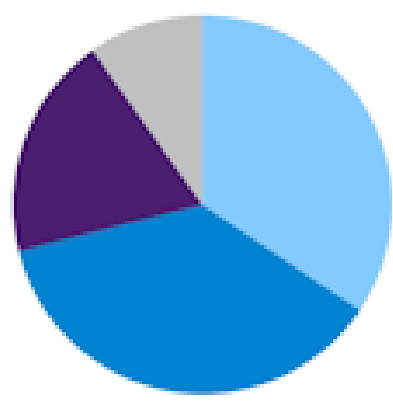

- transporter activity other 


\section{Average GC content in the three codon positions}

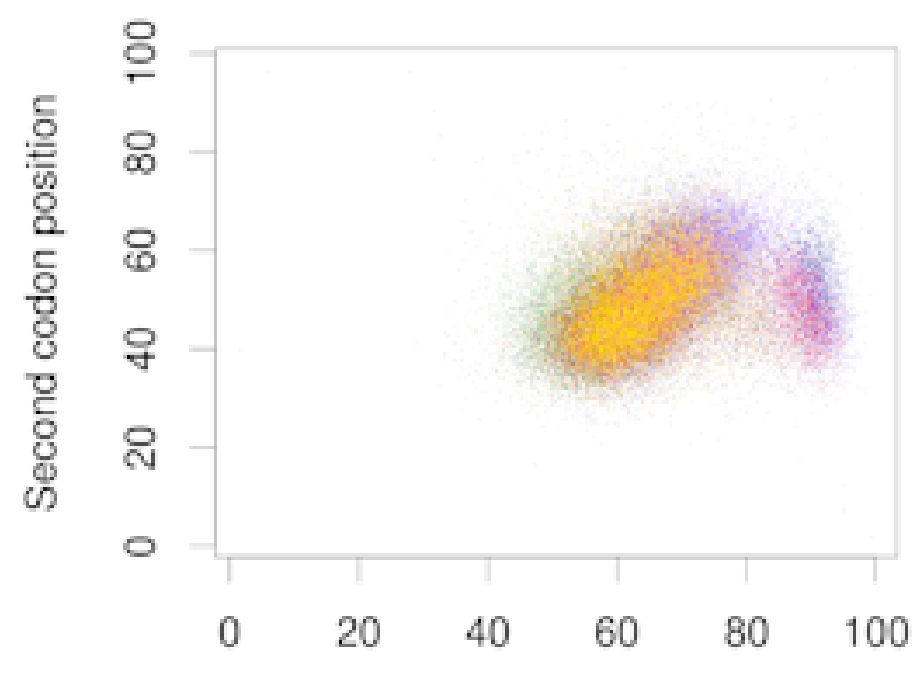

First codon position

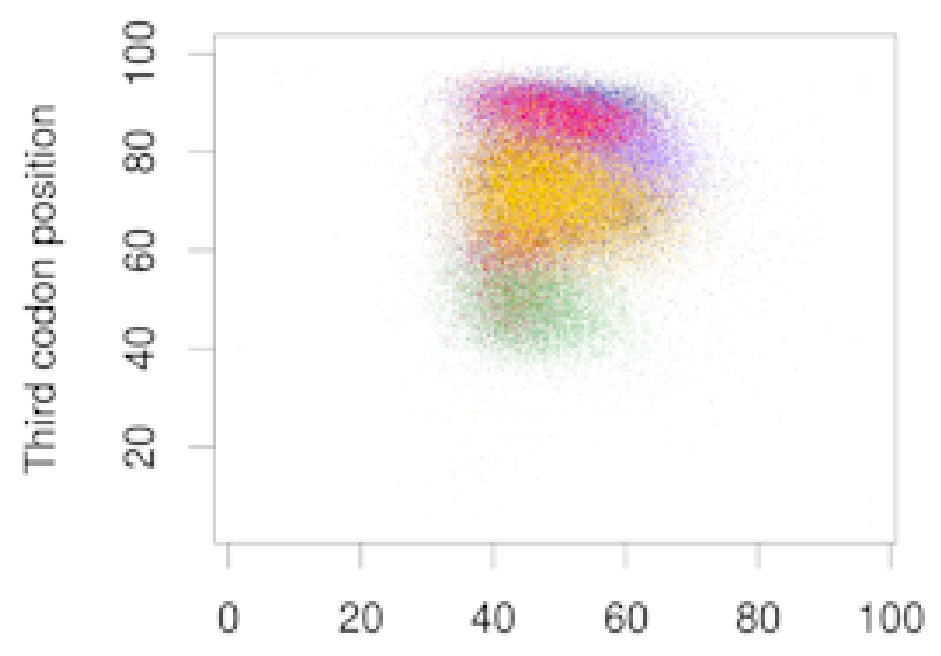

Second codon position

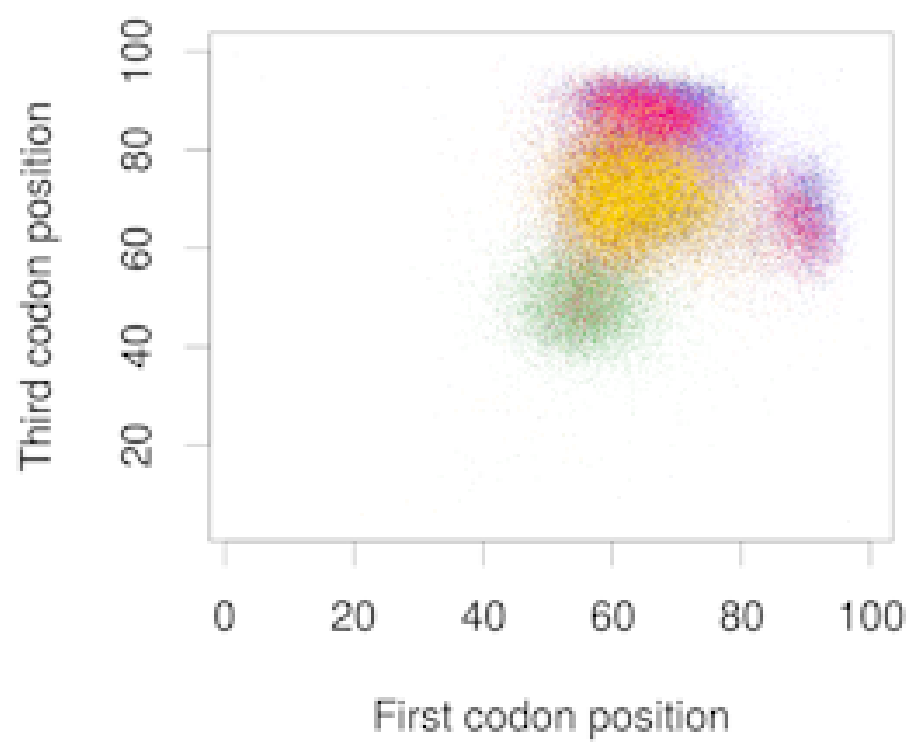

- Chlamydomonas acidophila

- Chlamydomonas reinhardtii

- Coccomyxa subellipsoidea

- Dunaliella acidophila

- Micromonas pusilla

- Micromonas sp. RC299

- Ostreococcus lucimarinus

- Volvox carteri 
Additional File 4. Simplified phylogenetic analyses of additional transcripts coding for genes with an annotation related to heavy metal tolerance and present in $C$. acidophila but not in C. reinhardtii. The phylograms represent the majority consensus of trees sampled after stationarity in the Bayesian analysis. PP values equal or greater than 0.50 are shown above branches. The scale bar indicates relative distance between different sequences based on mutation rate. A) ACR3 comp_14907 and comp_15936 B) MATE efflux protein comp_12911 C) NRAMP family comp_13602 D) ABC-ATPase comp_14042 E) arsenite-antimonite efflux family comp_15332 F) glutathione-regulated potassium-efflux comp_16013 G) multidrug efflux transporter AcrB comp_16471 H) multidrug resistance-associated protein comp_17557.

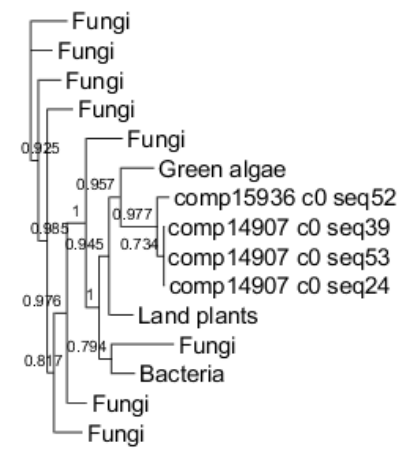

A) ACR3 comp_14907 and comp_15936

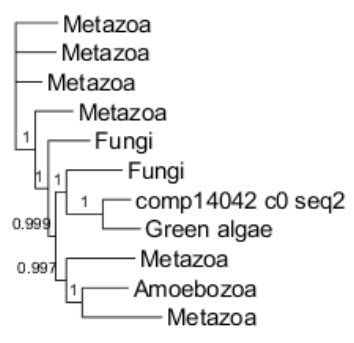

D) ABC-ATPase comp_14042

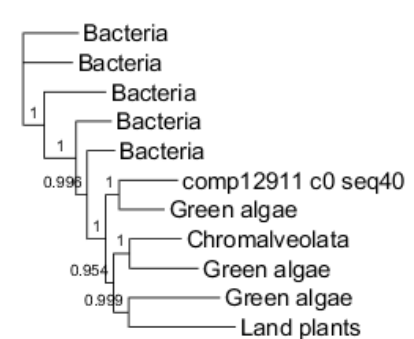

B) MATE efflux protein comp_12911

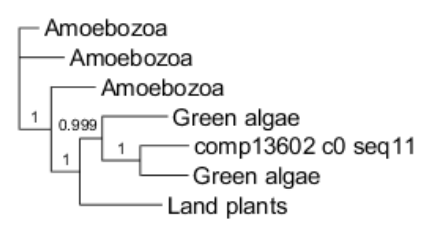

C) NRAMP family comp_13602

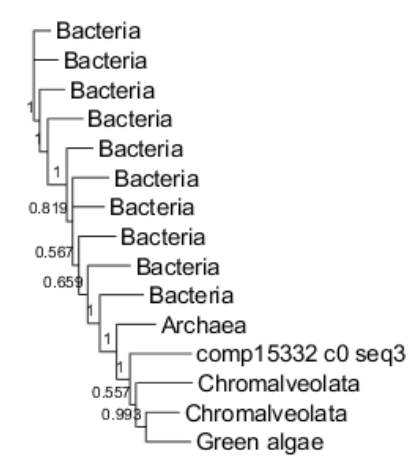

E) arsenite-antimonite efflux family comp_15332

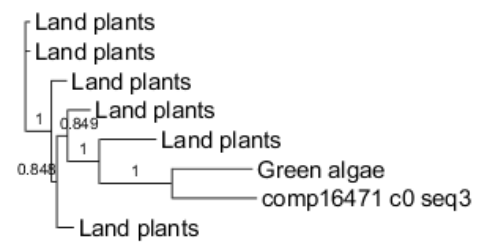

G) multidrug efflux transporter AcrB comp 16471

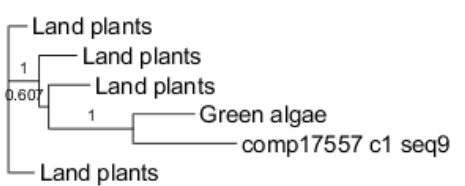

H) multidrug resistance-associated protein comp_17557 
